\title{
Bimetric, conformal supergravity and its superstring embedding
}

\author{
Sergio Ferrara, ${ }^{a, b, c}$ Alex Kehagias ${ }^{d}$ and Dieter Lüst ${ }^{e, f}$ \\ ${ }^{a}$ CERN, Theory Department, \\ 1211 Geneva 23, Switzerland \\ ${ }^{b}$ INFN - Laboratori Nazionali di Frascati, \\ Via Enrico Fermi 40, 00044 Frascati, Italy \\ ${ }^{c}$ Department of Physics and Astronomy and Mani L. Bhaumik Institute for Theoretical Physics, \\ U.C.L.A. Los Angeles CA 90095-1547, U.S.A. \\ ${ }^{d}$ Physics Division, National Technical University of Athens, \\ 15780 Zografou Campus, Athens, Greece \\ ${ }^{e}$ Arnold-Sommerfeld-Center for Theoretical Physics, \\ Ludwig-Maximilians-Universität, 80333 München, Germany \\ ${ }^{f}$ Max-Planck-Institut für Physik, Werner-Heisenberg-Institut, \\ 80805 München, Germany \\ E-mail: Sergio.Ferrara@cern.ch, kehagias@central.ntua.gr, \\ dieter.luest@lmu.de
}

ABSTRACT: We discuss the connection between $\mathrm{Weyl}^{2}$ supergravity and superstrings and further discuss holography between 4 -dimensional, $\mathcal{N}=4$ superconformal Weyl ${ }^{2}$ supergravity and $\mathcal{N}=8$, higher spin-four theory on $A d S_{5}$. The Weyl ${ }^{2}$ plus Einstein supergravity theory is a special kind of a bimetric gravity theory and consists of a massless graviton multiplet plus an additional massive spin-two supermultiplet. Here, we argue that the additional spin-two field and its superpartners originate from massive excitations in the open string sector; just like the $\mathcal{N}=4$ super Yang-Mills gauge fields, they are localized on the world volume of D3-branes. The ghost structure of the Weyl action should be considered as an artifact of the truncation of the infinitely many higher derivative terms underlying the massive spin 2 action. In field theory, $\mathcal{N}=4 \mathrm{Weyl}^{2}$ supergravity exhibits superconformal invariance in the limit of vanishing Planck mass. In string theory the additional spin-two fields become massless in the tensionless limit. Therefore low string scale scenarios with large extra dimensions provide (almost) superconformal field theories with almost massless open string spin-two fields. The full $\mathcal{N}=4$ scalar potential including the Yang-Mills matter multiplets is presented and the supersymmetric vacua of Einstein Supergravity are shown, as expected, to be vacua of massive Weyl supergravity. Other vacua are expected 
to exist which are not vacua of Einstein supergravity. Finally, we identify certain spin-four operators on the 4-dimensional boundary theory that could be the holographic duals of spin-four fields in the bulk.

KeYwords: AdS-CFT Correspondence, Conformal Field Models in String Theory, Dbranes, Superstring Vacua

ARXIV EPRINT: 1810.08147 


\section{Contents}

1 Introduction 1

2 Field theory: (super)-Yang-Mills plus (super)-Weyl gravity 3

2.1 Bosonic case 3

2.2 Supersymmetric case 5

2.3 Including gauge fields 6

$2.4 \mathcal{N}=4$ super-Yang-Mills plus super-Weyl theory 9

2.4.1 Massive theory 9

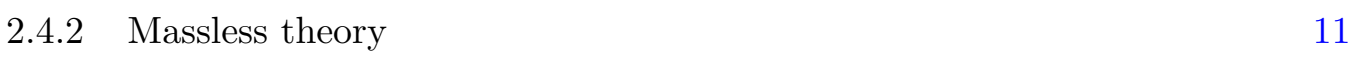

$\begin{array}{lll}2.4 .3 & \text { Scalar potential } & 13\end{array}$

3 String realization $\quad \mathbf{1 6}$

$\begin{array}{lll}3.1 & \text { Open string sector } & 16\end{array}$

$\begin{array}{lll}3.1 .1 & \text { Massless open string Yang-Mills sector } & 16\end{array}$

$\begin{array}{ll}\text { 3.1.2 Massive open string spin-two sector } & 17\end{array}$

$\begin{array}{lll}3.2 & \text { Closed string sector } & 19\end{array}$

$\begin{array}{lll}3.2 .1 & \text { Massless gravity sector } & 19\end{array}$

$\begin{array}{ll}\text { 3.2.2 Massive closed string spin-four sector } & 19\end{array}$

3.3 Effective field theory and limits 20

3.3.1 Ten-dimensional picture, non-compact space 20

3.3.2 Four-dimensional picture, compact internal space 22

4 Holographic aspects between spin-two on the boundary and spin-four in the bulk

5 Conclusions

\section{Introduction}

It is well known that the effective action of string theory is given in terms Einstein gravity, coupled to matter fields plus in finite series of higher derivative terms, which in particular contain an infinite series of higher curvature terms, which are suppressed by appropriate powers of the string scale $M_{s}=\left(\alpha^{\prime}\right)^{-1}$. In the so-called field theory limit of sending $\alpha^{\prime} \rightarrow 0$, all higher string modes decouple and all higher derivative interactions disappear, and the effective theory is just given by the Einstein-Yang-Mills-theory. Particular string examples of those theories are brane-world models, where the Yang-Mills degrees of freedom are localized on the world-volumes of stack of D-branes, and where the gravitational fields, namely the metric field $g_{\mu \nu}$ and its partners, correspond to closed strings, which propagate 
within the entire ten-dimensional bulk space. Here will will consider the simplest case, namely a stack of N D3-branes, i.e. the open string Yang-Mills sector is confined on the $4 \mathrm{D}$ world-volume of the D3-branes.

Now, when considering also higher curvature terms up to four derivatives [1-10], it is again well known that the $R^{2}$ action and the so-called Weyl ${ }^{2}$ action propagate additional degrees of freedom: for $R^{2}$ there is an additional scalar mode and for $\mathrm{Weyl}^{2}$ there exist an additional spin two field, denoted by $w_{\mu \nu}$. In this paper we will discuss the physics connected to the Weyl ${ }^{2}$ action and to spin-two field $w_{\mu \nu}$ and in particular the question how do they arise in string theory. Since the theory contains two spin-two metric fields, namely $g_{\mu \nu}$ and $w_{\mu \nu}$, it is a particular example of a bimetric gravity theory [11-15]. As we will discuss the second spin-two mode $w_{\mu \nu}$ is not contained in standard closed string gravitational sector, but it corresponds to the first massive open string excitations, namely to the massive excitations of the open string Yang-Mills gauge fields. Therefore these massive fields $w_{\mu \nu}$ are also localized on the world volume of D3-branes, and the effective $\mathrm{Weyl}^{2}$ is an entirely four-dimensional action on the world-volume of the D3-branes. As we will discuss, performing a particular scaling limit, and one is left with an effective 4D theory with one massless spin-two field plus one (almost) massless spin-two field, namely massless $\mathcal{N}=4$ super Yang-Mills gauge theory plus (almost) massless $\mathcal{N}=4$ super-Weyl $^{2}$ theory, whose spectrum was recently constructed in [16]. Hence in this limit the theory becomes (almost) superconformal invariant. Note that superconformal Weyl ${ }^{2}$ gravity [17-21], only exists for numbers of supersymmetries $\mathcal{N} \leq 4$, just like superconformal Yang-Mills gauge theories also only exist for $\mathcal{N} \leq 4$ [22]. This fact confirms our observation that Weyl ${ }^{2}$ gravity is not originating entirely from closed strings, but is an effective open string theory, localized on D3-branes.

These theories are also of phenomenological interest, namely in the context of the low string scale scenario together with large extra dimensions, which allows for unique predictions for the production of the massive open string excitations at particle physics collider machines [23]. Namely, following the discussion of this paper, the low string scale scenario with light, open string spin-two excitations is a (almost) superconformally invariant field theory.

As we will argue in the last part of the paper, the 4D (almost) super-conformal invariant Weyl supergravity theory allows for an holographic description in terms of closed string modes in an $A d S_{5}$ bulk theory. In contrast to the standard AdS/CFT correspondence between massless open string Yang-Mills gauge theory in the 4D boundary and supergravity in the 5D bulk, the holographic description of the (almost) massless spin-two fields on the boundary is given by (almost) massless spin-four fields in the higher-dimensional bulk.

The structure of the paper is as follows: in section 2 we describe Weyl supergravity coupled to super Yang-Mills theory. In section 3, we present a string theory realization of the theory and in section 4 we present some of its holographic aspects. Finally, section 5 contains our conclusions. 


\section{Field theory: (super)-Yang-Mills plus (super)-Weyl gravity}

\subsection{Bosonic case}

The most general formulation of Einstein plus curvature-square gravity is described by an action containing the standard Einstein term plus the following two terms being second order in the curvature tensor: ${ }^{1}$

$$
S=\int_{\mathcal{M}} d^{4} x \sqrt{-g}\left(M_{P}^{2} R+c_{1} W_{\mu \nu \rho \sigma} W^{\mu \nu \rho \sigma}+c_{2} R^{2}\right) .
$$

More details can be e.g. found in $[24,25]$. The first term with $W_{\mu \nu \rho \sigma}$ being the Weyl tensor

$$
W_{\mu \nu \rho \sigma}=R_{\mu \nu \rho \sigma}+g_{\mu[\sigma} R_{\rho] \nu}+g_{\nu[\rho} R_{\sigma] \mu}+\frac{R}{3} g_{\mu[\rho} g_{\sigma] \nu}
$$

is conformally invariant, whereas the $R^{2}$ term is only scale invariant. Indeed, the conformal transformation

$$
g_{\mu \nu} \rightarrow \widehat{g}_{\mu \nu}=\Omega^{2} g_{\mu \nu}
$$

leaves the Weyl tensor inert

$$
\widehat{W}_{\nu \rho \sigma}^{\mu}=W_{\nu \rho \sigma}^{\mu},
$$

whereas the curvature scalar transforms as

$$
\widehat{R}=\Omega^{-2} R-6 \Omega^{-3} g^{\mu \nu} \nabla_{\mu} \nabla_{\nu} \Omega .
$$

The two couplings $c_{i}$ in (2.1) are dimensionless. As discussed in [24], the $R^{2}$ action only propagates a scalar mode in flat four-dimensional space-time $R^{1,3}$. Since we are in particular interested in spin-two fields and not to the additional scalar mode in the string spectrum, the $R^{2}$ action is not relevant for us, and we will set the coupling $c_{2}=0$. However, the action (2.1) with $c_{2}=0$ is not conformal invariant since the Einstein-term is not invariant under conformal transformations. Therefore the Einstein-term can be regarded as the mass term in this theory, i.e. a mass deformation, which explicitly breaks conformal invariance.

The propagator of the Einstein-Weyl ${ }^{2}$ theory $[1,2]$ described by

$$
S=\int_{\mathcal{M}} d^{4} x \sqrt{-g}\left(M_{P}^{2} R+\frac{1}{2 g_{W}^{2}} W_{\mu \nu \rho \sigma} W^{\mu \nu \rho \sigma}\right)
$$

is given by the following expression

$$
\Delta_{\mu \nu \rho \sigma}=\Delta(k) P_{\mu \nu \rho \sigma},
$$

where

$$
\Delta(k)=\frac{g_{W}^{2}}{k^{2}\left(k^{2}-g_{W}^{2} M_{P}^{2}\right)},
$$

\footnotetext{
${ }^{1}$ There are two more linear combination of quadratic curvature terms, namely the Gauss-Bonnet and the Hirzebruch-Pontryagin action. However in four-dimensions these are total derivatives and hence we neglect them in the following. Similar considerations exist also in the supersymmetric case [26-29].
} 
and

$$
P_{\mu \nu \rho \sigma}=\frac{1}{2}\left(\theta_{\mu \rho} \theta_{\nu \sigma}+\theta_{\mu \sigma} \theta_{\nu \rho}\right)-\frac{1}{3} \theta_{\mu \nu} \theta_{\rho \sigma}
$$

with

$$
\theta_{\mu \nu}=\eta_{\mu \nu}-\frac{k_{\mu} k_{\nu}}{k^{2}}
$$

the usual transverse vector projection operator. Note that the propagator (2.7) for $M_{P}^{2}=0$ (i.e., pure Weyl ${ }^{2}$ theory) exhibits the conformal $1 / k^{4}$ behaviour. When the Einstein terms is present, we can equivalently write $\Delta(k)$ as

$$
\Delta(k)=-\frac{1}{M_{P}^{2}} \frac{1}{k^{2}}+\frac{1}{M_{P}^{2}} \frac{1}{k^{2}-g_{W}^{2} M_{P}^{2}},
$$

where the massless helicity- \pm 2 graviton is easily identified in the first term of (2.11). Moreover, we see that there is also a massive spin-2 state (the second term in (2.11)) with mass given by the pole at $k^{2}=g_{W}^{2} M_{P}^{2}$ which however has opposite residue to the usual massles graviton, and therefore describes a ghost spin-2 state. This shows that the theory contains as propagating degrees the standard, massless spin-two graviton $g_{\mu \nu}$ plus an additional massive spin-two field $w_{\mu \nu}$.

Actually, an alternative way to see this is to write down a particular bimetric gravity theory with two spin-two fields $g_{\mu \nu}$ and $w_{\mu \nu}$ with the following two-derivative action [30]:

$$
S=\int_{\mathcal{M}} d^{4} x \sqrt{-g}\left(M_{P}^{2} R(g)+2 M_{P} G_{\mu \nu}(g) w^{\mu \nu}-M_{W}^{2}\left(w^{\mu \nu} w_{\mu \nu}-a w^{2}\right)\right) .
$$

Here $G_{\mu \nu}=R_{\mu \nu}-1 / 2 R g_{\mu \nu}$ is the Einstein-tensor constructed from the metric $g_{\mu \nu}$ and the last term is a mass term for the second metric $w_{\mu \nu}$. In general the action propagates also a massive scalar mode. However setting the parameter $a=1$, the scalar mode disappears and the action contains a massless spin-two field $g_{\mu \nu}$ plus a massive spin-two field $w_{\mu \nu}$. Note that the two-derivative kinetic term for $w_{\mu \nu}$ is hidden in the coupling $G_{\mu \nu}(g) w^{\mu \nu}$, which can be seen by performing two partial integrations on this term. However after the partial integrations the kinetic term for $w_{\mu \nu}$ has the wrong sign, i.e. $w_{\mu \nu}$ is a ghost-like field. Now using the equation of motion

$$
\frac{\delta S}{\delta w_{\mu \nu}} \Rightarrow w_{\mu \nu}=\frac{M_{P}}{M_{W}^{2}}\left(R_{\mu \nu}(g)-\frac{1}{6} g_{\mu \nu} R\right),
$$

and plugging the solution for $w_{\mu \nu}$ back into the action (2.12), one can show [31] that the resulting action is (classically) equivalent to the four-derivative $W^{2}$ action in eq. (2.6) by using the fact that

$$
W_{\mu \nu \rho \sigma} W^{\mu \nu \rho \sigma}=G B+2\left(R_{\mu \nu} R^{\mu \nu}-\frac{1}{3} R^{2}\right),
$$

where $G B=R_{\mu \nu \rho \sigma} R^{\mu \nu \rho \sigma}-4 R_{\mu \nu} R^{\mu \nu}+R^{2}$ is the Gauss-Bonnet term. The bimetric gravity action (2.12) for $w_{\mu \nu}$ can be made ghost-free by adding an infinite number of terms with a 
finite number of parameters to it. As shown [31], this procedure is equivalent to adding to the $W^{2}$ action an infinite number of higher derivative terms, which resemble to additional parameters of the ghost-free bimetric gravity theory. In other words, the ghost nature of the massive spin-2 excitation is an artifact of the higher derivative truncation to fourth order. En passant, let us mention that for $a \neq 1$, the action (2.12) is (classically) equivalent to the action (2.1) with

$$
c_{1}=\frac{1}{2 g_{W}^{2}}, \quad c_{2}=\frac{a-1}{4 a-1} \frac{1}{3 g_{W}^{2}} .
$$

Therefore, only for $a=1$ the scalar mode associated to the $R^{2}$ term is absent.

\subsection{Supersymmetric case}

The above method can also be implemented in a supersymmetric setup [26-28]. For this, we need to recall that the graviton $h_{\mu \nu}$ sits in a real vector superfield $\Phi_{\mu}$ with expansion (in Wess-Zumino gauge)

$$
\Phi_{\mu}=\bar{\theta} \sigma^{\nu} \theta\left(h_{\mu \nu}+A_{\mu \nu}\right)+\frac{1}{2} \bar{\theta}^{2} \theta^{2} A_{\mu}+\cdots,
$$

where $A_{\mu \nu}$ and $A_{\mu}$ are the antisymmetric two-form and one-form fields of new-minimal supergravity, respectively. We can then define the real linear superfield $E_{\mu}$ as

$$
E^{\mu}=\frac{1}{2} \epsilon^{\mu \nu \rho \sigma} \bar{D} \sigma_{\nu} D \partial_{\rho} \Phi_{\sigma}
$$

which contains the Einstein term

$$
E^{\mu}=\bar{\theta} \sigma_{\nu} \theta\left(G^{\mu \nu}+\partial_{\lambda} F^{\lambda \nu \mu}+\frac{1}{2} \epsilon^{\nu \mu \rho \sigma} F_{\rho \sigma}\right)+\cdots,
$$

with $F_{\mu \nu \rho}=\partial_{\mu} A_{\nu \rho}+\partial_{\rho} A_{\mu \nu}+\partial_{\nu} A_{\rho \mu}$ and $F_{\mu \nu}=\partial_{\mu} A_{\nu}-\partial_{\nu} A_{\mu}$ the field strengths of the auxiliaries $A_{\mu \nu}$ and $A_{\mu}$, respectively. We need also to define the Riemann multiplet $R_{\mu \nu}$ with components expansion

$$
R_{\mu \nu}=\frac{1}{2} \psi_{\mu \nu}+\frac{i}{2} \theta^{2} \sigma^{\rho} \partial_{\rho} \bar{\psi}_{\mu \nu}-\frac{i}{2} \theta F_{\mu \nu}-\frac{i}{4} \sigma^{\kappa \lambda} \theta\left(R_{\kappa \lambda \mu \nu}+\partial_{\nu} F_{\mu \kappa \lambda}-\partial_{\mu} F_{\nu \kappa \lambda}\right) .
$$

The Weyl tensor $\mathcal{W}_{\mu \nu \rho \sigma}$ is contained then in the Weyl multiplet $W_{\mu \nu}$ defined as

$$
W_{\mu \nu}=\frac{1}{8}\left(\sigma^{\kappa \lambda} \sigma_{\mu \nu}+\frac{1}{3} \sigma_{\mu \nu} \sigma^{\kappa \lambda}\right) R_{\kappa \lambda}
$$

as can be seen from its components expansion

$$
W_{\mu \nu}=\frac{1}{16}\left(\sigma^{\kappa \lambda} \sigma_{\mu \nu}+\frac{1}{3} \sigma_{\mu \nu} \sigma^{\kappa \lambda}\right) \psi_{\kappa \lambda}-i \sigma^{\kappa \lambda} \theta W_{\kappa \lambda \mu \nu}+\cdots
$$

In terms of the real vector superfield $\Phi_{\mu}$, the Riemann and Weyl multiplets can be written (in spinor notation),

$$
R_{\mu \nu \alpha}=-\frac{1}{8} \bar{D}^{2} D_{\alpha}\left(\partial_{\mu} \Phi_{\nu}-\partial_{\nu} \Phi_{\mu}\right), \quad W_{\alpha \beta \gamma}=\frac{1}{16} \bar{D}^{2} D_{(\alpha} \partial_{\beta}^{\dot{\alpha}} \Phi_{\gamma) \dot{\alpha}}
$$


The action (2.1) (with $c_{2}=0$ ) is contained in the bosonic part of the supersymmetric Lagrangian (with $M_{P}=1$ here)

$$
\mathcal{L}=\int d^{4} \theta \Phi_{\mu} E^{\mu}+8 c_{1} \operatorname{Re} \int d^{2} \theta W_{\mu \nu} W^{\mu \nu} .
$$

The first term contains the Einstein term and the second the Weyl ${ }^{2}$. A supersymmetric generalization of (2.14) exists and it is written as

$$
W_{\mu \nu} W^{\mu \nu}=S G B-\frac{1}{8} \bar{D}^{2}\left(E_{\mu} E^{\mu}\right)+\frac{1}{3} W^{2},
$$

where

$$
W=\frac{1}{2} \sigma^{\mu} \bar{D} E_{\mu}
$$

and $S B G$ is the supersymmetric counterpart of the usual Gauss-Bonnet term and it is such that in the real and imaginary parts of its highest $\theta^{2}$ component are the HirzebruchPontryagin and Gauss-Bonnet terms, respectively. We may then write (2.24) as

$$
\mathcal{L}=\int d^{4} \theta\left(\Phi_{\mu} E^{\mu}-4 c_{1} E_{\mu} E^{\mu}\right)+\frac{8}{3} c_{1} \operatorname{Re} \int d^{2} \theta W^{2} .
$$

We may linearize in $E_{\mu}$ and $W$ the above Lagrangian by introducing a real vector superfield $V_{\mu}$ and a superfield $H$ so that

$$
\mathcal{L}=\int d^{4} \theta\left(\Phi_{\mu} E^{\mu}+2 V_{\mu} E^{\mu}+\frac{1}{4 c_{1}} V_{\mu} V^{\mu}\right)-\operatorname{Re} \int d^{2} \theta\left(2 W \bar{D}^{2} H+\frac{3}{8 c_{1}}\left(\bar{D}^{2} H\right)^{2}\right) .
$$

Then, after performing first the shift $\Phi_{\mu} \rightarrow \Phi_{\mu}-V_{\mu}$ and after the conformal transformation $\Phi_{\mu} \rightarrow \Phi_{\mu}+\bar{D} \sigma_{\mu} H+D \sigma_{\mu} \bar{H}$, we get that the supersymmetric action (2.26) is classically equivalent to

$$
\begin{aligned}
\mathcal{L}= & \int d^{4} \theta \Phi_{\mu} E^{\mu}-\int d^{2} \theta\left(V_{\mu} E^{\mu}-\frac{1}{4 c_{1}} V_{\mu} V^{\mu}+\frac{3}{8} L^{2}\right) \\
& -\operatorname{Re} \int d^{2} \theta\left(2 W \bar{D}^{2} H+\frac{3}{8 c_{1}}\left(\bar{D}^{2} H\right)^{2}\right),
\end{aligned}
$$

where $L=D \bar{D}^{2} H-\bar{D} D^{2} \bar{H}$ [26-28]. From the above Lagrangian we see that the first term in the first line describes a physical massless $\left(2, \frac{3}{2}\right)$ graviton multiplet $\left(\Phi_{\mu}\right)$, whereas the second term in the first line describes a massive $\left(2, \frac{3}{2}, \frac{3}{2}, 1\right)$ multiplet $\left(V_{\mu}\right)$ with mass square $m^{2}=1 / 4 c_{1}[32,33]$. The latter multiplet is not physical as its Lagrangian term opposite sign from the massless multiplet and therefore it is a ghost massive spin-2 multiplet.

\subsection{Including gauge fields}

Now, we will also include a four-dimensional bosonic Yang-Mills $\mathrm{U}(N)$ gauge theory, which is coupled to Einstein gravity. Then the action up to four orders in derivatives has the following form:

$$
S=\int d^{4} x \sqrt{-g}\left(-\frac{1}{4 g_{Y M}^{2}} F_{\mu \nu}^{a} F^{a \mu \nu}+\frac{1}{2 g_{W}^{2}} W_{\mu \nu \rho \sigma} W^{\mu \nu \rho \sigma}+M_{P}^{2} R\right) .
$$


$F_{\mu \nu}^{a}$ is the standard Yang-Mills field strength and $g_{W}^{2}$ and $g_{Y M}^{2}$ are dimensionless couplings. The Yang-Mills term and the $\mathrm{Weyl}^{2}$-term in the action possess (classical) conformal invariance, whereas again the Einstein-term can be regarded as the mass term in this theory.

Let us recall the propagating modes corresponding to this action. Specifically, there are three kinds of propagating modes $[1,2,32-35]$ :

(i) A massless helicity- \pm 2 graviton $g_{\mu \nu}$. This is the standard massless spin-two graviton.

(ii) Massless $\mathrm{U}(N)$ gauge bosons $A_{\mu}^{a}$.

(iii) A massive spin-two particle $w_{\mu \nu}$ with mass

$$
M_{W}=g_{W} M_{P}
$$

It is related to the $\mathrm{Weyl}^{2}$ term in the action. In fact as mentioned, this massive spin two particle is a ghost, destroying unitarity, but we will neglect this problem in the following and we will comment on it only in the conclusions. We will call this part of the spectrum the non-standard sector of the theory.

The Einstein plus (Weyl ${ }^{2}$ gravity theory contains seven propagating degrees of freedom. As already explained, this part of the theory can be considered as a bimetric theory of gravity with two spin-two fields, namely one the standard massless graviton $g_{\mu \nu}$ plus the non-standard massive spin-two field $w_{\mu \nu}$. As we will discuss in the following, in string theory the graviton $g_{\mu \nu}$ originates from the closed string sector and lives in the bulk space, whereas the spin-two field $w_{\mu \nu}$ as well as the Yang-Mills gauge bosons $A_{\mu}^{a}$ come from the open string sector and will be localized on the world-volume of a stack of D3-branes.

In the following we will consider the following three limits. Later we will see how these limits are realized in string theory.

(A) Decoupling of gravity, i.e. Yang-Mills limit. First we consider the infinite mass limit

$$
M_{P} \rightarrow \infty \text {. }
$$

In this limit gravity becomes non-dynamical and decouples from the theory. In fact, for non-zero coupling $g_{W}$, both spin-two particles completely decouple, since the spin-two particle $w_{\mu \nu}$ becomes infinitely heavy. Alternatively one can keep $M_{W}$ finite, which implies that $g_{W} \rightarrow 0$, i.e. the spin-two Weyl modes are very weakly coupled.

(B) Massless bigravity limit. Second we consider the massless limit, namely the limit of vanishing Planck mass: ${ }^{2}$

$$
M_{P} \rightarrow 0 .
$$

The propagator $\Delta(k)$ now becomes

$$
\Delta(k) \rightarrow \frac{g_{W}^{2}}{k^{4}}
$$

\footnotetext{
${ }^{2}$ The massless limit was also discussed in the context of bimetric theories in [31, 36].
} 
In this limit the second spin-two field $w_{\mu \nu}$ will become massless and we deal with massless Weyl gravity. Therefore, for finite $M_{P}$ there is a Higgs effect with respect to $w_{\mu \nu}$, and in the massless limit the degrees of freedom of $w_{\mu \nu}$ will arrange themselves into proper massless fields (see below). In this limit we deal with Yang-Mills gauge theory plus Weyl ${ }^{2}$ theory with action

$$
S=\int d^{4} x \sqrt{-g}\left(-\frac{1}{4 g_{Y M}^{2}} F_{\mu \nu}^{a} F^{a \mu \nu}+\frac{1}{2 g_{W}^{2}} W_{\mu \nu \rho \sigma} W^{\mu \nu \rho \sigma}\right) .
$$

This theory possesses conformal invariance and it propagates the following degrees of freedom:

(i) The standard massless, closed string spin-two graviton $g_{\mu \nu}$, corresponding to a planar wave in Einstein gravity.

(ii) Massless open string $\mathrm{U}(N)$ gauge bosons $A_{\mu}^{a}$.

(iii) In the non-standard sector there is massless open string spin-two ghost particle $w_{\mu \nu}$, which corresponds to a non-planar wave. In addition there is a massless open string vector $w_{\mu}$, which originates from the \pm 1 helicities of the massive $w_{\mu \nu}$ particle. However note that the helicity zero component of $w_{\mu \nu}$ does not correspond to a physical, propagating mode in the massless limit, since it can be gauged away by the conformal transformations (2.3).

(C) Light spin-two plus massless Yang-Mills limit. Now we consider the double scaling limit

$$
M_{P} \rightarrow \infty \quad \text { and } \quad g_{W} \rightarrow 0 \quad \text { with } \quad M_{W} \ll M_{P} .
$$

Therefore the coupling $g_{W}$ must vanish faster than $M_{P}^{-1}$. In this limit one is left which an action that contains the massless Yang-Mills gauge fields $A_{\mu}$ as well as the (almost) massless spin-two fields $w_{\mu \nu}$. In addition there is still the massless standard spin-two graviton. The propagator has the leading behaviour (2.33) and the dynamics is described again by the action (2.34). To see that this limit actually exists, one can recall the propagator in eq. (2.11) which for $M_{W}=g_{W} M_{P} \rightarrow 0$ can be approximated by

$$
\begin{aligned}
\Delta(k) & =-\frac{1}{M_{P}^{2}} \frac{1}{k^{2}}+\frac{1}{M_{P}^{2}} \frac{1}{k^{2}-M_{W}^{2}} \\
& \approx-\frac{1}{M_{P}^{2}} \frac{1}{k^{2}}+\frac{1}{M_{P}^{2}} \frac{1}{k^{2}}\left(1+\frac{M_{W}^{2}}{k^{2}}+\mathcal{O}\left(M_{W}^{4}\right)\right) \approx \frac{g_{W}^{2}}{k^{4}}+\mathcal{O}\left(M_{W}^{4}\right) .
\end{aligned}
$$

This is the propagator (2.33) for the massless Weyl ${ }^{2}$ theory and dynamics should indeed be described approximately by (2.34).

However, there is a difference between the case (B) and (C). In the case (B), the vanishing of the Einstein-Hilbert term, results in the action (2.34) with the propagator in (2.33). This is the standard propagator of a spin-2 dipole ghost. Indeed, the Lagrangian in this case in the linearized level will be of the form

$$
\mathcal{L}_{2} \sim \frac{1}{g_{W}^{2}}\left(\square h_{\mu \nu}\right)^{2}+h_{\mu \nu} T^{\mu \nu}
$$


where $h_{\mu \nu}$ is transverse-traceless and we have included its coupling to the energymomentum tensor. Then, one can introduce a transverse-traceless spin-2 field $\gamma_{\mu \nu}$ and write (2.37) in the form (after rescaling $h_{\mu \nu} \rightarrow g_{W} h_{\mu \nu}$ )

$$
\mathcal{L}_{2} \sim \gamma^{\mu \nu} \square h_{\mu \nu}-\frac{1}{4} \gamma_{\mu \nu} \gamma^{\mu \nu}+g_{W} h_{\mu \nu} T^{\mu \nu},
$$

typical of a dipole ghost $[32,33]$. Indeed, integrating out $\gamma_{\mu \nu}$ from (2.38), we get back $(2.37$. Can we identify one of the fields $\gamma_{\mu \nu}$ or $h_{\mu \nu}$ with the standard graviton? To answer this question one should recall that standard graviton is the field that couples to the energy-momentum tensor when it has the standard kinetic term. However, as one can see, although $h_{\mu \nu}$ couples to the energy-momentum tensor, it does not have standard kinetic term. Indeed, we can define fields $h_{ \pm \mu \nu}=\frac{1}{2}\left(h_{\mu \nu} \pm \gamma_{\mu \nu}\right)$ with diagonal kinetic term so that

$$
\mathcal{L}_{2} \sim h_{+\mu \nu} \square h_{+\mu \nu}-h_{-\mu \nu} \square h_{-\mu \nu}-\frac{1}{4}\left(h_{+\mu \nu}-h_{-\mu \nu}\right)^{2}+g_{W} h_{+\mu \nu} T^{\mu \nu}+g_{W} h_{-\mu \nu} T^{\mu \nu} .
$$

Clearly, both $h_{ \pm}$coupled to the energy-momentum tensor and there is no standard graviton.

In the case $(\mathrm{C})$ now, we have the linearized Lagrangian

$$
\begin{aligned}
\mathcal{L}_{2} & \sim M_{P}^{2} h_{\mu \nu} \square h^{\mu \nu}+\frac{1}{g_{W}^{2}}\left(\square h^{\mu \nu}\right)^{2}+h_{\mu \nu} T^{\mu \nu} \\
& \sim M_{P}^{2} h_{\mu \nu} \square h^{\mu \nu}+\frac{1}{g_{W}^{2}} \square h^{\mu \nu} \gamma^{\mu \nu}-\frac{1}{4 g_{W}^{2}} \gamma_{\mu \nu} \gamma^{\mu \nu}+h_{\mu \nu} T^{\mu \nu}
\end{aligned}
$$

or, after rescaling $h_{\mu \nu} \rightarrow h_{\mu \nu} / M_{P}$

$$
\mathcal{L}_{2} \sim h_{\mu \nu} \square h^{\mu \nu}+\frac{1}{M_{W}^{2}}\left(\square h^{\mu \nu}\right)^{2}+\frac{1}{M_{P}} h_{\mu \nu} T^{\mu \nu} .
$$

In the limit of very large $M_{P}$ and very small $g_{W}$ with fixed $M_{W}=g_{W} M_{P}$ as in the case (C), we have a light massive spin-2 (with mass $M_{W} \ll M_{P}$ ) and the standard massless graviton, although very weakly coupled now.

\section{$2.4 \mathcal{N}=4$ super-Yang-Mills plus super-Weyl theory}

\subsubsection{Massive theory}

Now let us come to the $\mathcal{N}=4$ supersymmetric version of the Einstein, Yang-Mills plus Weyl $^{2}$ theory. The spectrum of the $\mathcal{N}=4$ Super-Yang-Mills plus massive $\mathcal{N}=4$ SuperWeyl theory has the following form [16, 37]:

(i) A standard massless spin-two super graviton multiplet $g_{\mathcal{N}=4}$ with $n_{B}+n_{F}=32$ degrees of freedom and with the following helicities and SU(4) representations:

$$
(+2, \underline{1})+\left(+\frac{3}{2}, \underline{4}\right)+(1, \underline{6})+\left(+\frac{1}{2}, \underline{4}\right)+(0, \underline{1})
$$

together with its CPT conjugate

$$
(0, \underline{1})+\left(-\frac{1}{2}, \underline{4}\right)+(-1, \underline{6})+\left(-\frac{3}{2}, \overline{4}\right)+(-2, \underline{1}) .
$$

The complex scalar corresponds to the complex coupling constant $\tau$ of the $\mathcal{N}=4$ field theory, i.e. to the massless marginal operator in the superconformal field theory. 
(ii) A standard massless spin-one, $\mathcal{N}=4$ super Yang-Mills multiplet $W^{a}\left(a=1, \ldots, N^{2}\right)$ of the $\mathrm{U}(N)$ gauge group with each $n_{B}+n_{F}=16$ degrees of freedom and with the following helicities and $\mathrm{SU}(4)$ representations:

$$
(+1, \underline{1})+\left(+\frac{1}{2}, \underline{4}\right)+(0, \underline{6})+\left(-\frac{1}{2}, \overline{4}\right)+(-1, \underline{1}) .
$$

Here the $6 \times N$ scalars from the Cartan subalgebra superfields are additional marginal operators, which parametrize the Coulomb branch of the $\mathcal{N}=4$ super Yang-Mills gauge theory. Giving them generic vev's breaks the $\mathrm{U}(N)$ gauge symmetry to its maximal Abelian subgroup $\mathrm{U}(1)^{N}$. Together with the axion-dilaton field $\tau$ of the supergravity multiplet which couples to the quadratic YM action, these massless scalars parametrize the moduli space $\mathcal{M}$ of the theory which is given by the following coset space:

$$
\mathcal{M}=\frac{\mathrm{SU}(1,1)}{\mathrm{U}(1)} \otimes R^{6 N} .
$$

Note that the $6 N$ scalars $\Phi_{i j}=-\Phi^{j i},(i, j=1, \cdots, 4)$, of the $N$ vector multiplets are coupled to the curvature scalar in confrormal supergravity as

$$
\mathcal{L}=\cdots-\frac{1}{12} \operatorname{Tr}\left(\Phi_{i j} \Phi^{i j}\right)(R+\cdots)
$$

Therefore, the conditions

$$
\operatorname{Tr}\left(\Phi_{i j} \Phi^{i j}\right)=-6, \quad \operatorname{Tr}\left(\Phi_{i j} \psi^{j}\right)=0,
$$

where $\psi^{j}$ ate the gauginos, break superconformal dilatations and S-supersymmetry, lead to Poincaré supergravity and in this case the scalars parametrize the coset [38-41]

$$
\frac{\mathrm{SU}(1,1)}{\mathrm{U}(1)} \otimes \frac{\mathrm{SO}(6, N)}{\mathrm{SO}(6) \times \mathrm{SO}(N)}
$$

In fact, the conditions (2.47) are weaker than the constraints

$$
\operatorname{Tr}\left(\Phi_{i j} \Phi^{k l}\right)=-\frac{1}{2} \delta_{[i}^{k} \delta_{j]}^{l}, \quad \operatorname{Tr}\left(\Phi_{i j} \psi^{k}\right)=0,
$$

imposed by the equations of motion of the scalars $D^{i j}{ }_{k l}$ and the fermion $\chi^{i j}$, which we describe in section 2.4.3. These constraints allow to remove six vector multiplets in massless Einstein supergravity. Notice that in rigid supersymmetry, the Yang-Mills scalar manifold is flat $R^{6 N}$ whereas in Poincare supergravity the coset is $\mathrm{SO}(6, N) / \mathrm{SO}(6) \times \mathrm{SO}(N)$. It looks that in massive Weyl supergravity the scalar manifold is $\mathrm{SO}(6, N) / \mathrm{SO}(N)$ because 15 scalars have not been Higgsed. In other words the constraints (2.47) and (2.49) remove the 1 and 20 from $6 \times 6=1+20+15$ but do not remove the 15. The first constraint in (2.49) coming from the $D$ scalars which appear linearly in Einstein supergravity, is just a contribution to the scalar potential in massive Weyl supergravity because the $D$ scalars appear now quadratically in the Lagrangian. Hence, the deformation of (2.45) to (2.48) is only true if 
the Weyl term is absent so that the 15 gauge fields of the superconformal multiplet are auxiliary and their equations of motion produce the deformation from $R^{6 N}$ to $\mathrm{SO}(6, N) / \mathrm{SO}(6) \times \mathrm{SO}(N)$. However if the Weyl action term is added, the 15 vectors are massive and propagating and the above coset is not reproduced. Poincaré supergravity is the limit $M_{p} \rightarrow \infty$ while Weyl supergravity is the limit $M_{p}=0$. What happen in between is a new theory we are describing. The potential of this new theory is different from Poincaré supergravity and is strictly quartic in all scalar fields before imposing the constraints as we will see below.

(iii) In the non-standard sector we have the spin-two massive Weyl multiplet of $\mathcal{N}=4$, which is irreducible with $n_{B}+n_{F}=2^{8}=256$ states in USp(8) representations [37]:

$$
w_{\mathcal{N}=4}: \quad \operatorname{Spin}(2)+\underline{8} \times \operatorname{Spin}(3 / 2)+\underline{27} \times \operatorname{Spin}(1)+\underline{48} \times \operatorname{Spin}(1 / 2)+\underline{42} \times \operatorname{Spin}((2) .50)
$$

Hence in summary, the $\mathcal{N}=4$ massive super-(Weyl) $)^{2}$ gravity theory contains $n_{B}+$ $n_{F}=288+16 N$ degrees of freedom, where $N$ is the number of physical vector multiplets. General massive multiplets in extended supersymmetry were discussed in [43].

Also note that in Einstein supergravity constraints (2.47) and (2.49) are field constraints while in massive Weyl supergravity they are VEV constraints (Higgs phase) since the six vector multiplets, which appear in the massless limit (see next section) are in this case physical degrees of freedom. As we will now see, in massless Weyl supergravity these multiplets become unphysical gauge degrees of freedom since the massless Weyl action does not depend on compensators being superconformal invariant. So in massless Weyl supergravity coupled to Yang-Mills the moduli space is that in eq. (2.45). The massive phase is obtained when six extra singlet compensating vector multiplets are introduced.

\subsubsection{Massless theory}

Now we can consider the $\mathcal{N}=4$ supersymmetric version of the Higgs effect for the spin-two Weyl superfield $w_{\mathcal{N}=4}$. In the limit $M_{P} \rightarrow 0$ the bosonic and fermionic degrees of freedom of $w_{\mathcal{N}=4}$ will arrange themselves into proper massless supermultiplets, when taking into account the additional local superconformal and gauge symmetries, which arise in the massless limit. In order to perform the massless limit we need the branching rules of the massive USp(8) R-symmetry group into the R-symmetry group $\mathrm{SU}(4)$ of the massless states. The specific decomposition of $\mathrm{USp}(8) \rightarrow \mathrm{SU}(4)$ for the relevant representations is as follows:

$$
\begin{aligned}
\underline{8} & =\underline{4} \oplus \underline{\overline{4}}, \\
\underline{27} & =\underline{6} \oplus \underline{\overline{6}} \oplus \underline{15}, \\
\underline{42} & =\underline{1} \oplus \underline{\overline{1}} \oplus \underline{10}+\underline{10} \oplus \underline{20^{\prime}}, \\
\underline{48} & =\underline{20} \oplus \underline{\underline{20}} \oplus \underline{4} \oplus \underline{4}
\end{aligned}
$$

Then for $M_{P}=0$, the spectrum of the massless $\mathcal{N}=4$ Super-Weyl theory has the following form [16]: 
(i) A standard massless spin-two supergravity multiplet with $n_{B}+n_{F}=32$ degrees of freedom as given in eqs. (2.42) and (2.43).

(ii) In the non-standard sector, we get first from the massive Weyl multiplet $w_{\mathcal{N}=4}$ a massless ghost-like spin-two supermultiplet with $n_{B}+n_{F}=32$ and with the helicites and $\mathrm{SU}(4)$ quantum numbers, again as given eqs. (2.42) and (2.43).

Second we get from $w_{\mathcal{N}=4}$ four massless spin-3/2 supermultiplets (in total $n_{B}+n_{F}=$ 128 ) with the following helicities and SU(4) representations, namely

$$
\underline{\overline{4}} \times\left[\left(\frac{3}{2}, \underline{1}\right)+(1, \underline{4})+\left(\frac{1}{2}, \underline{6}\right)+(0, \underline{\overline{4}})+\left(-\frac{1}{2}, \underline{1}\right)\right],
$$

together with the CPT conjugate states

$$
\underline{4} \times\left[\left(\frac{1}{2}, \underline{1}\right)+(0, \underline{4})+\left(-\frac{1}{2}, \underline{6}\right)+(-1, \underline{\overline{4}})+\left(-\frac{3}{2}, \underline{1}\right)\right] .
$$

They contain the 15 gauge bosons of the local $\mathrm{SU}(4)_{R}$ gauge symmetry.

In addition, the massive Weyl multiplet $w_{\mathcal{N}=4}$ contains $\operatorname{six} \mathcal{N}=4$ vector multiplets of the form:

$$
6(\text { spin - one }): \underline{6} \times\left[(+1, \underline{1})+\left(+\frac{1}{2}, \underline{4}\right)+(0, \underline{6})+\left(-\frac{1}{2}, \overline{4}\right)+(-1, \underline{1}) .\right]
$$

However these multiplets are unphysical since they can be gauged away by the superconformal transformations together with the local $\mathrm{SU}(4)_{R}$ transformations. Specifically, one of the 36 scalars in these vector multiplets is a Weyl mode. Other 15 scalars are the helicity zero component of the massive vectors inside $w_{\mathcal{N}=4}$, which are gauged away by the local $\mathrm{SU}(4)_{R}$ transformations. Hence all six vector-multiplets are unphysical, do not propagate and get removed from the spectrum.

We should note that the dipole ghost graviton and the tripole ghost spin-3/2 sector are accompanied by a dipole ghost complex scalar since the action is a higher-derivative action. Indeed, the equations of motion are fourth-order for the spin- 2 and third order for the spin-3/2 states. This fact is also discussed in [44] at the Lagrangian level. This is not the case for the SU(4) gauge bosons which have standard Yang Mills action. The sugra higher derivative action also contains a singlet vector mode which, together with the gauge bosons, is part of the higher derivative gravitino action (which as pointed out above obeys third order equations of motion). In other words, the cubic gravitino action simultaneously describes the gravitino, the partner of the graviton, as well as the gravitini of the gravitino multiplet.

Hence, the massless $\mathcal{N}=4$ super-(Weyl) ${ }^{2}$ gravity theory contains $n_{B}+n_{F}=192$ physical, propagating degrees of freedom. The same spectrum was also obtained in [42] using the string twistor formalism for the construction of $\mathcal{N}=4$ super-(Weyl) ${ }^{2}$ gravity. The spin $1 / 2$ have three sources, from the spin $3 / 2$ cubic gravitino kinetic term, the spin $1 / 2$ cubic kinetic term and the spin $1 / 2$ standard Majorana kinetic term. 


\begin{tabular}{|cccc|}
\hline Scalars & SU(4) rep. & w & c \\
\hline \hline$\phi^{\alpha}$ & $\underline{1}$ & 0 & 1 \\
$E_{i j}$ & $\underline{10}$ & 1 & -1 \\
$D^{i j}{ }_{k l}$ & $\underline{20}$ & 2 & 0 \\
$\Phi_{i j}$ & $\underline{6}$ & 1 & 0 \\
\hline
\end{tabular}

Table 1. Scalars of the Weyl multiplet $(\phi, E, D)$ and the Yang-Mills multiplet $(\Phi)$, together with their SU(4) assignments, Weyl $(w)$ and chiral $(c)$ weights.

At the end of this section, we can summarize the spectrum of Weyl supergravity in the following way. In pure Weyl supergravity without any additional massless Yang-Mills multiplets, the six vector multiplets with $36=1+15+20$ helicity zero components play the role of super-goldstone bosons. In the massless conformal Weyl phase $\left(M_{P} \rightarrow 0\right)$ the six compensators are not there and the spectrum goes from 256 massive +32 massless states to $160+32=192$ massless states. The $160=32+128$ massless states correspond to the second graviton multiplet plus four gravitini multiplets. On the contrary if we delete the Weyl square part and we keep the six compensator vector multiplets we have the constraints (2.41) and (2.43), and we get back massless spin-two Einstein supergravity.

\subsubsection{Scalar potential}

In this section we will consider some couplings between the Yang-Mills sector and the Weyl sector of the theory. In particular we will discuss the potential of the scalar fields that appear in the $\mathcal{N}=4$ Yang-Mills and Weyl supermultiplets. The scalar fields of the Weyl and the Yang-Mills multiplet of the $\mathcal{N}=4$ conformal supergravity ${ }^{3}$ coupled to super Yang-Mills transform under specific representations of SU(4) which are tabulated in table 1, where also their Weyl weights and chiral $\mathrm{U}(1)$ weights $w$ and $c$, respectively are given [37, 39-41]. The indices $i, j, \ldots$ and $a, b, \ldots$ are $\mathrm{SU}(4)$ and $\mathrm{SU}(1,1)$ indices, respectively. In particular, $\phi^{\alpha}$ represent two-degrees of freedom associated to the $\mathrm{SU}(1,1) / \mathrm{U}(1)$ coset of the spin-two dipole ghost multiplet, $E_{i j}$ is symmetric, $D^{i j}{ }_{k l}$ is pseudoreal and $\Phi_{i j}$ is antisymmetric, and in the adjoint representation of the gauge group $G$. They satisfy the relations

$$
\phi^{\alpha} \phi_{\alpha}=1, \quad E_{i j}=E_{j i}, \quad D_{k l}^{i j}=\frac{1}{4} \epsilon_{m n}^{i j} \epsilon_{k l}^{p q} D_{p q}^{m n}, \quad D_{k j}^{i j}=0, \quad \Phi_{i j}=-\Phi_{j i},
$$

whereas their complex conjugate fields are

$$
\begin{aligned}
& \phi_{1}=\left(\phi^{1}\right)^{*}, \\
& \phi_{2}=-\left(\phi^{2}\right)^{*}, \\
& E^{i j}=\left(E_{i j}\right)^{*}, \\
& D_{i j}^{k l}=\left(D^{i j}{ }_{k l}\right)^{*}=D_{i j}^{k l} \text {, } \\
& \Phi^{i j}=\left(\Phi_{i j}\right)^{*}=-\frac{1}{2} \epsilon^{i j k l} \Phi_{k l} .
\end{aligned}
$$

Notice that in eq. (2.50) we have seen that the spin-two massive Weyl multiplet of $\mathcal{N}=4$ in the non-standard sector has $n_{B}+n_{F}=2^{8}=256$ states which are arranged in $\operatorname{USp}(8)$

\footnotetext{
${ }^{3}$ We use freely the terms Weyl and conformal supergravity in an interchangable way, and similalry for the terms Einstein and Poincaré supergravity.
} 
representations as follows

$$
\operatorname{Spin}(2)+\underline{8} \times \operatorname{Spin}(3 / 2)+\underline{27} \times \operatorname{Spin}(1)+\underline{48} \times \operatorname{Spin}(1 / 2)+\underline{42} \times \operatorname{Spin}(0) .
$$

Therefore the scalars in the massive multiplet are in the $\underline{42}$ representation of $\operatorname{USp}(8)$. The latter is decomposed under $\mathrm{SU}(4) \subset \mathrm{USp}(8)$ as

$$
\underline{42}=\underline{20}+\underline{10}+\underline{\overline{10}}+\underline{1}+\underline{\overline{1}},
$$

and it is associated to the pseudoreal $D_{i j}{ }^{k l}(\underline{20})$, the complex $E_{i j}(\underline{10}+\underline{10})$ and the complex $\phi^{\alpha}(\underline{1}+\underline{\overline{1}})$ of table 1 . The six scalars $\Phi_{i j}\left(=-\Phi_{j i}\right)$ in the $\underline{6}$ of $\mathrm{SU}(4)$ and in the adjoint of the gauge group are just the scalars of the Yang-Mills multiplet. Note that the fields $D_{i j}{ }^{k l}(\underline{20})$, which appear in the unphysical vector multiplets in eq. (2.54), are unphysical in the massless limit. Moreover the scalars in the $\underline{6}+\underline{\overline{6}}$ representations of the spin- $3 / 2$ multiplets (see eqs. (2.52) and (2.53)) are not part of the scalar potential, because they originate from the graviphoton fields.

The most general Lagrangian for the $\mathcal{N}=4$ conformal supergravity has been constructed in [41]. It turns out that it is completely specified by a single holomorphic and homogeneous of zeroth degree function $\mathcal{H}\left(\phi_{\alpha}\right)$ of the coset variables $\phi_{\alpha}$. Then the bosonic part of the Weyl square action has a field dependent coupling constant $1 / g_{W}^{2}=\simeq \Re\left(\mathcal{H}\left(\phi_{\alpha}\right)\right)$ which is of the form of conformal supergravity in twistor-string theory [42]:

$$
\mathcal{L}_{W^{2}} \simeq \Re\left(\mathcal{H}\left(\phi_{\alpha}\right)\right) W_{\mu \nu \rho \sigma} W^{\mu \nu \rho \sigma} .
$$

The structure of the scalar potential for $\mathcal{N}=4$ super Yang-Mills is coupled to $\mathcal{N}=4$ conformal supergravity can be read off from refs [39-41] and it turns out to be (in the notation of [41])

$$
\begin{aligned}
V= & \mathcal{H}\left(\frac{1}{8} D^{i j}{ }_{k l} D^{k l}{ }_{i j}-\frac{1}{16} E_{i j} E^{j k} E_{k l} E^{l i}+\frac{1}{48}\left(E_{i j} E^{i j}\right)^{2}\right)+\frac{1}{16} \mathcal{D H} D^{i j}{ }_{k l} E_{i m} E_{j n} \epsilon^{k l m n} \\
& +\frac{1}{384} \mathcal{D}^{2} \mathcal{H} E_{i j} E_{k l} E_{m n} E_{p q} \epsilon^{i k m p} \epsilon^{j l n q}-\frac{1}{48} E_{i j} E^{i j} \operatorname{Tr}\left(\Phi_{k l} \Phi^{k l}\right) \\
& +\frac{1}{8} D^{i j}{ }_{k l} \operatorname{Tr}\left(\Phi_{i j} \Phi^{k l}\right)+\frac{1}{3} \bar{f}(\phi) E^{i j} \operatorname{Tr}\left(\Phi^{k l}\left[\Phi_{i k}, \Phi_{j l}\right]\right) \\
& +\frac{1}{4}|f(\phi)|^{2} \operatorname{Tr}\left(\left[\Phi_{i k}, \Phi^{k j}\right]\left[\Phi_{j l}, \Phi^{l i}\right]\right)+\text { h.c. }
\end{aligned}
$$

where $\mathcal{D}$ is the operator

$$
\mathcal{D}=-\phi^{\alpha} \epsilon_{\alpha \beta} \frac{\partial}{\partial \phi_{\beta}}, \quad \text { and } \quad f(\phi)=\phi^{1}+\phi^{2} .
$$

In rigid supersymmetry, only the last term of the potential (2.60) exists. All the other terms arise from the Weyl multiplet (terms proportional to $\mathcal{H}$ and its derivatives) and the gauge-matter coupling. Note also that with the $\mathrm{U}(1)$ charge $c$ assignment $c(\mathcal{H})=0$, $c(\mathcal{D H})=2$ and $c\left(\mathcal{D}^{2} \mathcal{H}\right)=4$, the potential $(2.60)$ is $\mathrm{U}(1)$ invariant $(c(V)=0)$ since $c(E)=-1, c(D)=c(\Phi)=0$ and $c\left(\phi^{\alpha}\right)=1$. Therefore the potential in eq. (2.60) is what 
we would call "massless Weyl supergravity coupled to matter" whose massive Poincaré supergravity deformation is obtained by adding six compensator vector multiplets with constraints given as in eq. (2.47).

The scalars $D^{i j} k$ are auxiliaries and can be integrated out leading to

$$
\begin{aligned}
V= & \mathcal{H}\left(-\frac{1}{16} E_{i j} E^{j k} E_{k l} E^{l i}+\frac{1}{48}\left(E_{i j} E^{i j}\right)^{2}\right)-\frac{1}{128 \mathcal{H}}\left(\mathcal{D H} E_{i m} E_{j n} \epsilon^{k l m n}+2 \operatorname{Tr}\left(\Phi_{i j}^{k l}\right)\right)^{2} \\
& +\frac{1}{384} \mathcal{D}^{2} \mathcal{H} E_{i j} E_{k l} E_{m n} E_{p q} \epsilon^{i k m p} \epsilon^{j l n q}-\frac{1}{48} E_{i j} E^{i j} \operatorname{Tr}\left(\Phi_{k l} \Phi^{k l}\right) \\
& +\frac{1}{3} \bar{f}(\phi) E^{i j} \operatorname{Tr}\left(\Phi^{k l}\left[\Phi_{i k}, \Phi_{j l}\right]\right)+\frac{1}{4}|f(\phi)|^{2} \operatorname{Tr}\left(\left[\Phi_{i k}, \Phi^{k j}\right]\left[\Phi_{j l}, \Phi^{l i}\right]\right)+\text { h.c. },
\end{aligned}
$$

where

$$
\Phi_{k l}^{i j}=\Phi^{i j} \Phi_{k l}-2 \delta_{[l}^{[j} \Phi^{i] m} \Phi_{k] m}+\frac{1}{3} \delta_{[k}^{i} \delta_{l]}^{j} \Phi^{p q} \Phi_{p q} .
$$

Note that a non-constant $\mathcal{H}$ function gives extra terms to the scalar potential (2.62). This will be the case in twistor string theory where $\mathcal{H}$ is an exponential in the holomorphic variable [42]. For constant $\mathcal{H}$, the terms proportional to $\mathcal{D H}$ and $\mathcal{D}^{2} \mathcal{H}$ in the potential drop and it is easy to see that $E=0$ and $\Phi$ in the Cartan subalgebra of the gauge group is an extremum of the potential. This is the breaking of superconformal to Poincare supergravity if 6 auxiliary vector multiplets are added with wrong sign so that a correct Einstein term and the solution $D=0$ is possible. Indeed, let us recall that the fermions of the theory are the gravitini $\psi_{\mu}^{i}$ (in the 4 of SU(4)) associated with Q-supersymmetry, the composite $\phi_{\mu i}$ (in the $\overline{4}$ ) associated with S-supersymmetry and the two spinor fields $\Lambda_{i}$ and $\chi^{i j}{ }_{k}$ in the $\overline{4}$ and 20 of SU(4), respectively. The fermionic shifts of the spinors fields contain among others, the terms [37]

$$
\begin{aligned}
\delta \Lambda_{i} & =\cdots+E_{i j} \epsilon^{j} \\
\delta \chi_{k}^{i j} & =\cdots+D_{k l}^{i j} \epsilon^{l}-\frac{1}{2} \epsilon^{i j l m} E_{k l} \eta_{m},
\end{aligned}
$$

where $\epsilon^{i}$ and $\eta^{i}$ are the Q- and S-supersymmetry parameters. Therefore, $E=0$ and $D=0$ are the necessary conditions for unbroken supersymmetry. In addition, for Poincaré supersymmetry, breaking of Weyl symmetry is required. This is achieved by imposing the condition (2.47) while still $E=D=0$. If there are non-trivial extrema of the scalar potential beyond the supersymmetric Poincaré one is an interesting open problem. Such vacua will further break Poincaré supersymmetry, which will happen if the $E$ and $D$ scalars have non-vanishing vev.

We note that pure massive Weyl supergravity is obtained by adding to the Weyl multiplet 6 vector multiplets of wrong sign. In this case the spectrum is the standard massless $\mathcal{N}=4$ Poincaré supergravity coupled to a massive $\mathcal{N}=4$ spin-2 ghost multiplet. The massive scalars are then 20 from the six compensators, the $10+\overline{10} E$ scalars and $1+1$ from the dilaton dipole massive ghost. All together they make the 42 (of USp(8)) as it should. Indeed, the constraint (2.49) is needed in Poincare supergravity because the $D$ scalars appear linearly in the action [39, 40]. However, this is not true in Weyl massive 
supergravity where they appear quadratically [41] so that they lead to a new potential term after integrate them out rather than to a constraint. In the higgsed phase, the 15 scalars go away and this explains $42=1+1+20+10+\overline{10}$.

\section{String realization}

Now we want to discuss how to obtain $\mathrm{Weyl}^{2}$ gravity plus Yang-Mills gauge theory from IIB superstring theory. As already mentioned, in string theory the graviton $g_{\mu \nu}$ originates from the closed string sector and lives in the bulk space, whereas the spin-two field $w_{\mu \nu}$ as well as the Yang-Mills gauge bosons $A_{\mu}^{a}$ come from the open string sector and will be localized on the world-volume of a stack of D3-branes. In the following we will first discuss the closed and open string spectrum and then, how the various limits can be realized in string theory.

Here we will discuss the case of maximal supersymmetry. This means that in fourdimensional the closed string bulk theory possesses $\mathcal{N}=8$ supersymmetry (i.e. 32 supercharges), whereas the open string sector localized on the D-brane worldvolume will preserve $\mathcal{N}=4$ supersymmetry (i.e. 16 supercharges). Specifically, we will consider the type IIB superstring on $R^{1,3} \times T^{6}$, with an additional stack of $\mathrm{N}$ D3-branes with world-volumes on $R^{1,3}$. Possible other D-branes and/or orientifold planes do not play an important role for the discussion, and we also do not address the question of tadpole cancellation. In fact, when taking the decoupling limit of infinite $T^{6}$ volume later on, i.e. considering a non-compact six-dimensional extra space, we just deal with N D3-branes in flat tendimensional space-time.

The spectrum of this string theory is now as follows.

\subsection{Open string sector}

\subsubsection{Massless open string Yang-Mills sector}

Now we come to the massless open string spectrum of the D3-branes on the background $R^{1,3} \times T^{6}$. For maximally supersymmetric, toroidal compactifications of $D=10$ superstring, its excitations form supermultiplets of $\mathcal{N}=4$ supersymmetry. Before discussing the first excited level, we recall the vertices of massless particles, which arise from the zero modes and include, in the NS sector, the gauge bosons $A^{a}$ and six real scalars $\phi^{I}, I=1, \ldots, 6$. In the $\mathrm{R}$ sector, we have four gauginos $\lambda^{A}, I=A, \ldots, 4$. All in all, these zero mode form one $\mathcal{N}=4$ gauge supermultiplet. The NS sector vertices, in the $(-1)$-ghost picture, read:

$$
\begin{aligned}
V_{A^{a}}^{(-1)}(z, \epsilon, k) & =g_{A} T^{a} e^{-\phi} \epsilon^{\mu} \psi_{\mu} e^{i k X}, \\
V_{\phi^{a}, I}^{(-1)}(z, k) & =g_{A} T^{a} e^{-\phi} \Psi^{I} e^{i k X} .
\end{aligned}
$$

Here, $X, \psi, Z, \Psi$ are the fields of $\mathcal{N}=1$ worldsheet SCFT, with the Greek indices associated to $D=4$ spacetime fields $X^{\mu}, \psi^{\nu}$ and the Latin upper case labeling internal $D=6$ (e.g. $\left.Z^{I}, \Psi^{I}\right) . \phi$ is the scalar bosonizing the superghost system. 
The $\mathrm{R}$ sector vertices, in the $(-1 / 2)$-ghost picture, read:

$$
\begin{aligned}
& V_{\lambda^{a, A}}^{(-1 / 2)}(z, u, k)=g_{\lambda} T^{a} e^{-\phi / 2} u^{\sigma} S_{\sigma} \Sigma^{A} e^{i k X}, \\
& V_{\bar{\lambda}^{a, A}}^{(-1 / 2)}(z, \bar{u}, k)=g_{\lambda} T^{a} e^{-\phi / 2} \bar{u}_{\dot{\sigma}} \bar{S}^{\dot{\sigma}} \bar{\Sigma}^{A} e^{i k X} .
\end{aligned}
$$

Here, $S$ and $\bar{S}$ are the left and right-handed SU(2) spin fields, respectively, while $\Sigma^{A}$ and $\bar{\Sigma}^{A}$ are the internal Ramond spin fields. The couplings are

$$
g_{A}=\left(2 \alpha^{\prime}\right)^{1 / 2} g_{Y M} \quad, \quad g_{\lambda}=\left(2 \alpha^{\prime}\right)^{1 / 2} \alpha^{1 / 4} g_{Y M},
$$

where $g_{Y M}$ is the gauge coupling. In the above definitions, $T^{a}$ are the Chan-Paton factors accounting for the gauge degrees of freedom of the two open string ends, meaning that all these massless states are in the adjoint representation of the $\mathrm{U}(N)$ gauge group.

We can also write these states in terms of the fermionic oscillators in the transversal space-time directions, denoted by $b_{r}^{i}(i=1,2)$, and the internal oscillators $b_{r}^{I}(I=1, \ldots, 6)$. Then the eight bosons bosonic states in he adjoint representation look like

$$
A_{i}^{a} \sim T^{a} b_{-1 / 2}^{i}|0\rangle, \quad \Phi^{a, I} \sim T^{a} b_{-1 / 2}^{I}|0\rangle
$$

For the eight fermions in the adjoint representation one simply has

$$
\lambda^{a, A} \sim T^{a}|\dot{\alpha}, A\rangle,
$$

where $|\dot{\alpha}, A\rangle$ is the Ramond ground state with four-dimensional spinor-helicity index $\dot{\alpha}=$ 1,2 and internal spinor index $A=1, \ldots, 4$. These states indeed built massless $\mathcal{N}=4$ vector multiplets in the adjoint representation of the gauge group $\mathrm{U}(N)$, which are displayed in eq. (2.44). They are localized at the world-volume of the N D3-branes.

\subsubsection{Massive open string spin-two sector}

We will now determine the first excited, massive open string states, which are also localized at the world-volume of the N D3-branes. For maximally supersymmetric, toroidal compactifications of $D=10$ superstring, NS and R sectors form one spin-two massive supermultiplet of $\mathcal{N}=4$ supersymmetry. The bosons form one symmetric tensor field $B_{m n}$ and one completely antisymmetric tensor field $E_{m n p}$. Here, the indices $(m, n, p)$ label $D=10$. All these particles are in the adjoint representation of the gauge group. The corresponding vertices, in the $(-1)$-ghost picture, read [45]:

$$
V_{N S, a}^{(-1)}(z, k)=\frac{g_{A}}{\sqrt{2 \alpha^{\prime}}} T^{a} e^{-\phi}\left(E_{m n p} \psi^{m} \psi^{n} \psi^{p}+B_{m n} i \partial X^{m} \psi^{n}+H_{m} \partial \psi^{m}\right) e^{i k X},
$$

where $H_{m}$ is an auxiliary vector field. Note that again the open string gauge coupling $g_{A}=\left(2 \alpha^{\prime}\right)^{1 / 2} g_{Y M}$ appears in this vertex operator. At this level, the on-shell condition is $k^{2}=-\frac{1}{\alpha^{\prime}}$. The constraints due to the requirement of BRS invariance are:

$$
\begin{aligned}
k^{m} E_{m n p} & =0, \\
2 \alpha^{\prime} k^{m} B_{m n}+H_{n} & =0, \\
B_{m}^{m}+k^{m} H_{m} & =0 .
\end{aligned}
$$


In $D=10$ all 128 bosonic degrees of freedom can be accounted for by setting $H=0$, i.e. with a traceless, transverse $B$ and transverse $E$.

Also for the fermions, we begin with the first massive level in $D=10$. In the $\mathrm{R}$ sector, the fermion vertex operator [in its canonical $(-1 / 2)$-ghost picture] is parametrized by two vectors, Majorana-Weyl spinors $v_{m}^{A}$ and $\bar{\rho}_{\dot{B}}^{n}$ of opposite chirality [45]:

$$
V_{R, a}^{(-1 / 2)}(z, v, \bar{\rho}, k)=C_{\Lambda} T^{a}\left[v_{m}^{A} i \partial X^{m}+2 \alpha^{\prime} \bar{\rho}_{\dot{B}}^{m} \psi_{m} \psi^{n} \Gamma_{n}^{\dot{B} A}\right] \Theta_{A} e^{-\phi / 2} e^{i k X} .
$$

Here, $A$ denotes a left-handed spinor index while $\dot{B}$ is its right handed counterpart. $\Gamma_{n}$ are $16 \times 16$ Weyl blocks of the $D=10$ gamma matrices and $\Theta_{A}$ are the conformal weight $h=\frac{5}{8}$ chiral spin fields.

Requiring BRST invariance imposes two on-shell constraints on $v_{m}^{A}$ and $\bar{\rho}_{\dot{B}}^{m}$ which determine $\bar{\rho}$ in terms of $v$ and leave 144 independent components in the latter. Furthermore, a set of 16 spurious states exists which allows to take $\bar{\rho}$ and $v$ as transverse and $\Gamma$-traceless:

$$
k^{m} v_{m}^{A}=v_{m}^{A} \Gamma_{A \dot{B}}^{m}=k_{m} \bar{\rho}_{\dot{B}}^{m}=\bar{\rho}_{\dot{B}}^{m} \Gamma_{m}^{\dot{B} A}=0 .
$$

These $128=144-16$ physical degrees of freedom match the counting for bosons.

As for the massless states, we can also write the massive states, that are created by these vertex operators, in terms of the bosonic and fermionic oscillators $\alpha_{n}$ and $b_{r}$. Now we split the indices into uncompactified and internal indices. Furthermore we will omit the gauge index, i.e. we drop the Chan-Paton factor $T^{a}$, which means that we consider the neutral, excited states of the Abelian $\mathrm{U}(1)$ vector-multiplet. This $\mathrm{U}(1)$ gauge group is just the Abelian part of the full gauge group $\mathrm{U}(N)=\mathrm{SU}(N) \times \mathrm{U}(1)$. Alternatively we could consider the case of a single D3-brane, i.e. $N=1$, where the excited states are also neutral. Then one obtains at the first massive level the following massive open string states (see for example [46]):

$$
\begin{aligned}
& b_{-1 / 2}^{i} b_{-1 / 2}^{j} b_{-1 / 2}^{I}|0\rangle, \quad b_{-1 / 2}^{i} b_{-1 / 2}^{I} b_{-1 / 2}^{J}|0\rangle, \quad b_{-1 / 2}^{I} b_{-1 / 2}^{J} b_{-1 / 2}^{K}|0\rangle, \\
& b_{-3 / 2}^{i}|0\rangle, \quad b_{-3 / 2}^{I}|0\rangle \\
& \alpha_{-1}^{i} b_{-1 / 2}^{j}|0\rangle, \quad \alpha_{-1}^{i} b_{-1 / 2}^{I}|0\rangle, \quad \alpha_{-1}^{I} b_{-1 / 2}^{i}|0\rangle, \quad \alpha_{-1}^{I} b_{-1 / 2}^{J}|0\rangle .
\end{aligned}
$$

(Here the $b$ 's and the $\alpha$ 's are the oscillators of the world-sheet fermions and bosons.) Collecting all states and putting them into proper massive representations of the fourdimensional little group $\mathrm{SO}(3)$ as well as in proper representations of the $\mathcal{N}=4 \mathrm{SU}(4)$ R-symmetry, one obtains the following massive spectrum:

$$
\underline{1} \times \operatorname{Spin}(2)+(\underline{6}+\underline{6}+\underline{15}) \times \operatorname{Spin}(1)+\left(2 \times \underline{1}+\underline{10}+\underline{10}+\underline{20^{\prime}}\right) \times \operatorname{Spin}(0) .
$$

For massive states in $\mathcal{N}=4$ supersymmetry the R-symmetry group is enhanced from $\mathrm{U}(4)$ to $\mathrm{USp}(8) \supset \mathrm{U}(4)$ with the following branching rules:

$$
\begin{aligned}
\underline{8} & =\underline{4}+\underline{\overline{4}}, \\
\underline{27} & =\underline{6}+\underline{6}+\underline{15}, \\
\underline{36} & =\underline{1}+\underline{10}+\underline{10}+\underline{15}, \\
\underline{42} & =2 \times \underline{1}+\underline{10}+\underline{10}+\underline{20^{\prime}}, \\
\underline{48} & =\underline{4}+\underline{\overline{4}}+\underline{20}+\underline{20}
\end{aligned}
$$


Then the massive bosons transform under $\operatorname{USp}(8)$ as

$$
\underline{1} \times \operatorname{Spin}(2)+(\underline{27}) \times \operatorname{Spin}(1)+(\underline{42}) \times \operatorname{Spin}(0) .
$$

In ten dimensions, the 128 massive fermions are given by the following string states:

$$
(8)_{c}+(56)_{c}: \quad b_{-1}^{A}|a\rangle, \quad(8)_{s}+(56)_{s}: \quad \alpha_{-1}^{A}|\dot{a}\rangle .
$$

In terms of four-dimensional massive spinors this leads to:

$$
(\underline{4}+\underline{\overline{4}}) \times \operatorname{Spin}(3 / 2)+(\underline{4}+\underline{\overline{4}}+\underline{20}+\underline{20}) \times \operatorname{Spin}(1 / 2),
$$

where in this decomposition each spin 3/2 Rarita Schwinger field in four dimensions contains 4 degrees of freedom and each spin 1/2 Dirac fermion possess 2 degrees of freedom. Under USp(8) the massive fermions transform as

$$
(\underline{8}) \times \operatorname{Spin}(3 / 2)+(\underline{48}) \times \operatorname{Spin}(1 / 2),
$$

The bosons in eq. (3.11) together with the fermions in eq. (3.16) build one long, massive $\mathcal{N}=4$ spin 2 supermultiplet. It precisely agrees with the super Weyl multiplet $w_{\mathcal{N}=4}$, which is displayed in eq. (2.50).

\subsection{Closed string sector}

In the following we will also provide the closed string spectrum of the theory, both in the bulk and also on the stack of the D3-branes. The vertex operators are similar to one of the open strings and obtained by the tensor product of left- and right-moving open string states at each mass level, taking into account the level matching constraint $h_{L}=h_{R}$.

\subsubsection{Massless gravity sector}

Let us us first recall the closed string type II B spectrum of the bulk theory on the background space $R^{1,3} \times T^{6}$. As it is well known, the massless closed string states originate from the (NS,NS), (R,R), (R,NS) and (NS,R) sectors of the theory. Altogether they built the standard massless $\mathcal{N}=8$ supergravity multiplet with $n_{B}+n_{F}=256$ propagating massless degrees of freedom. However on the world volume of the stack of N D3-branes supersymmetry is broken by half from $\mathcal{N}=8$ to $\mathcal{N}=4$, where 16 supersymmetries are linearly realized and the other half of 16 supersymmetries are non-linearly realized on the D3-branes. Therefore the massless closed string spectrum on the D3-branes is precisely the one of $\mathcal{N}=4$ supergravity. The corresponding massless states precisely build the standard massless spin-two super graviton multiplet $g_{\mathcal{N}=4}$, which is displayed in eqs. (2.42) and (2.43).

\subsubsection{Massive closed string spin-four sector}

As discussed in [47], the first excited closed string states are obtained by performing the tensor product of two super-Weyl supermultiplets. This leads to a massive supermultiplet with a highest spin-four tensor field $\Phi^{4}$ in the closed string sector, whereas the massive spintwo sector, i.e. the massive Weyl supermultiplets, correspond to open string excitations. 
For the case under consideration with background space $R^{1,3} \times T^{6}$, the bulk spectrum is then given in terms of massive spin-four $\mathcal{N}=8$ supermultiplet $\Phi_{\mathcal{N}=8}^{4}$ :

$$
\Phi_{\mathcal{N}=8}^{4}=w_{\mathcal{N}=4} \otimes w_{\mathcal{N}=4}
$$

It contains $n_{B}+n_{F}=256 \times 256=10^{16}=65.536$ degrees of freedom. When restricting it to the world volume of the N D3-branes, it gets truncated and becomes massive spinfour $\mathcal{N}=4$ supermultiplet $\Phi_{\mathcal{N}=4}^{4}$ with $n_{B}+n_{F}=1280$. Its exact multiplet structure is as follows:

$$
\begin{aligned}
\underline{1} \times \operatorname{Spin}(4)+\underline{8} \times \operatorname{Spin}(7 / 2)+(\underline{1}+\underline{27}) \times \operatorname{Spin}(3)+(\underline{8}+\underline{48}) \times \operatorname{Spin}(5 / 2) \\
\quad+(\underline{1}+\underline{27}+\underline{42}) \times \operatorname{Spin}(2)+(\underline{8}+\underline{48}) \times \operatorname{Spin}(3 / 2)+(\underline{1}+\underline{27}) \times \operatorname{Spin}(1) \\
\quad+\underline{8} \times \operatorname{Spin}(1 / 2)+\underline{1} \times \operatorname{Spin}(0) .
\end{aligned}
$$

\subsection{Effective field theory and limits}

Now we will discuss the four-dimensional effective field theory on the stack of N D3 branes. From the closed strings we will restrict ourselves to the massless gravitational sector, and the closed string spin-four in the bulk will be mentioned later in the next section on holography. For the open strings, we will consider the massless spin-one Yang-Mills sector as well as on the massive spin-two Weyl sector. Since both types of fields belong to open string with ends lying on the D3-branes, the Yang-Mills field as well as the Weyl fields are confined to the world-volumes of the D3-branes.

\subsubsection{Ten-dimensional picture, non-compact space}

Here we consider a stack of N D3-branes in a non-compact space $R^{1,9}$. The ten-dimensional action can be schematically written as

$$
S=S_{\text {bulk }}+S_{\text {brane }}+S_{\text {int }}
$$

where $S_{\text {bulk }}$ is the effective action of the massless gravitons and their superpartners from the closed strings, $S_{\text {brane }}$ is the four-dimensional effective action of the massless Yang-Mills fields and the massive spin-two field $w_{\mu \nu}$ on the D3-branes,

$$
S_{\text {brane }}=S_{Y M}+S_{W}
$$

and $S_{\text {int }}$ describes the interactions between the open and closed string modes.

Let us now determine the effective couplings in terms of the basic string parameters, which are

(i) $g_{s}=e^{\phi}$, the string coupling constant, which is determined by the vev of the dilaton and

(ii) $M_{s}=1 / \sqrt{\alpha^{\prime}}$, namely the string scale. 
In the string frame, the effective ten-dimensional Planck mass is given as

$$
\kappa^{(10)}=\left(M_{P}^{(10)}\right)^{-4}=\frac{1}{M_{s}^{4}} g_{s} .
$$

The masses $M_{n}$ of the string excitations in the string frame directly follow from the fundamental string tension and are given by $M_{n}^{2}=n M_{s}^{2}$. Namely in the string scale the mass $M_{W}$ of the first open string excitations is simply given as

$$
M_{W}=M_{s} .
$$

In order to go to the Einstein frame, one has to perform a Weyl rescaling of the metric, which in $\mathrm{D}$ dimensions takes the form

$$
\begin{aligned}
g & \rightarrow \exp (\phi / 2) g \\
\sqrt{|g|}_{D} & \rightarrow \exp (D \phi / 4) \sqrt{|g|}_{D}, \\
R & \rightarrow \exp (-\phi / 2) R
\end{aligned}
$$

(Hence for $D=4$ the Weyl action $W^{2} \sqrt{|g|}$ is indeed invariant under this rescaling.)

Therefore the ten-dimensional Einstein-Hilbert term transforms from the string frame into the Einstein frame as

$$
\sqrt{|g|}_{10} e^{-2 \phi} R \rightarrow \sqrt{|g|}_{10} R
$$

and in the Einstein frame the Planck mass is therefore independent of $g_{s}$ :

$$
\kappa^{(10)}=\left(M_{P}^{(10)}\right)^{-4}=\frac{1}{M_{s}^{4}} .
$$

Second, the gauge kinetic term of a Dp-brane transforms from the string frame into the Einstein frame as

$$
\sqrt{|g|}_{p+1} e^{-\phi} F^{\mu \nu} F_{\mu \nu} \rightarrow e^{((p-7) / 4 \phi)} \sqrt{|g|}_{p+1} F^{\mu \nu} F_{\mu \nu}
$$

Hence, for D3-branes $(p=3)$ the effective gauge coupling in the Einstein frame is given as

$$
g_{\mathrm{YM}}=\sqrt{g_{s}} .
$$

Finally for the fundamental string tension one obtains that

$$
\sqrt{|g|}_{1+1} \rightarrow e^{\phi / 2} \sqrt{|g|}_{1+1}
$$

Therefore the masses of the excited strings in the Einstein frame scale as

$$
M_{n}^{2} \sim n \sqrt{g_{s}} M_{s}^{2},
$$

and hence the ratio between these masses and the 10d Planck scale remains invariant. In D dimensions, a mass, when measured in the Einstein metric, is related to $g_{s}$ as

$$
M_{n}^{2} \sim n g_{s}^{\frac{4}{D-2}} M_{s}^{2} .
$$


In the limit $\alpha^{\prime}=M_{s}^{-2} \rightarrow 0$, while keeping $g_{s}, N$ and all other physical length scales, such as curvature scales fixed, all massive string excitations decouple and the higher derivative interactions can be neglected. Furthermore, open and closed string modes decouple and gravity becomes free, i.e. we arrive at a theory of free gravitons and its supersymmetry partners. This decoupling limit is also referred to the Maldacena limit: free type IIB supergravity in the bulk and four-dimensional SYM theory with 16 supercharges on the world-volume of the branes. To see the more precise form of the decoupling limit, which zooms into the near horizon region of the D3-brane SUGRA solution, we recall that it is defined as follows:

$$
L M_{s} \rightarrow \infty \quad \text { with } \quad L^{4}=\frac{g_{s} N}{M_{s}^{4}} .
$$

On the gauge theory side this limit corresponds to the limit of infinite 't Hooft coupling

$$
\lambda \rightarrow \infty \text { with } \lambda=g_{\mathrm{YM}}^{2} N
$$

In this the near-horizon limit the type IIB background of the N D3 branes is given by the well-known $A d S_{5} \times S^{5}$ geometry. Note that this limit can be obtained by sending $L$ to infinity while keeping $M_{s}$ fixed, which means that the near horizon limit can be obtained for finite masses of the string excitations.

\subsubsection{Four-dimensional picture, compact internal space}

Now we switch to four dimensions and consider the theory compactified on $R^{1,3} \times T^{6}$. As we have discussed in section 3.1.2 the massive open string excitations precisely agree with the $\mathcal{N}=4$ spin-two Weyl supermultiplet. The question is now, which is the correct effective action for these massive states. Since these states appear at the first mass level, the corresponding effective action must contain four derivatives. Hence a priori, it could be either the $R^{2}$-action or the $W^{2}$-action. Since the $R^{2}$ propagates a scalar degree of freedom, whereas the $W^{2}$-action propagates precisely the spin-two degrees of freedom of the Weyl-supermultiplet, we can safely conclude that the $W^{2}$-action is the correct effective action for the massive open string fields. Therefore the four-dimensional string effective active action for closed string gravity plus open string Yang-Mills plus open string massive spin-two fields has the following form:

$$
S_{\mathrm{eff}}=\int d^{4} x \sqrt{-g}\left(-\frac{1}{4 g_{\mathrm{YM}}^{2}} F_{\mu \nu}^{a} F^{a \mu \nu}+\frac{1}{2 g_{W}^{2}} W_{\mu \nu \rho \sigma} W^{\mu \nu \rho \sigma}+M_{P}^{2} R\right) .
$$

In addition to the ten-dimensional string parameters $g_{s}$ and $M_{s}$ we now gain a third parameter, namely:

(iii) $R_{i}$ : the radius of the internal space, i.e. the volume of the $T^{6}$ is given by $R_{i}^{6}$. In units of the string length $L_{s}$ the size of the internal scape is given by the dimensionless parameter $r=R_{i} / L_{s}=R_{i} M_{s}$.

The three string parameters $g_{s}, M_{s}$ and $r$ are identified with the three four-dimensional coupling constants of the effective theory in the following way: 
(i) The four-dimensional gravitational closed string coupling $M_{P}$ in the Einstein frame:

$$
M_{P}=M_{s} r^{3}
$$

(ii) The open string Yang-Mills coupling $g_{\mathrm{YM}}$ for the gauge fields on the D3-branes:

$$
g_{\mathrm{YM}}=\sqrt{g_{s}} .
$$

(iii) The bimetric Weyl coupling $g_{W}$ : the effective $4 \mathrm{D}$ coupling $g_{W}$ can be determined by the requirement that the mass of the massive open string spin-two fields $w_{\mu \nu}$ is given in the Einstein frame as (see eq. (3.30)))

$$
M_{W}=g_{s} M_{s} .
$$

It then follows from eq. (2.30) that

$$
g_{W}=g_{s} / r^{3} .
$$

Observe that in the four-dimensional Einstein frame, the Weyl coupling $g_{W}$ is scaling with respect to $g_{s}$ as the gravitational coupling, because it corresponds to a coupling between closed and open strings. Moreover is proportional to the inverse of the internal volume.

Now we can consider the following four decoupling limits in the four-dimensional effective string theory, which we already mentioned before in section two:

(A) Decoupling of gravity. The decoupling of the closed string modes namely the decoupling of standard gravity is achieved sending the Planck mass to infinity:

$$
M_{P} \rightarrow \infty \text {. }
$$

In this limit either the string scale $M_{s}$ is very large, i.e. $\alpha^{\prime} \rightarrow 0$ with $r$ kept fixed. Alternatively one can keep $M_{s}$ finite, but sending $r \rightarrow \infty$, implying that $R_{i} \gg L_{s}$ and the internal space becomes very large. Then the near horizon geometry close to the N D3-branes becomes $A d S_{5} \times S^{5}$. In this sense the size $R_{i}$ of the internal space corresponds to the length parameter $L$ in the non-compact case. Both, for finite $r$ and large $M_{s}$ and also for large $r$ and finite $M_{s}$ the massive spin-two open string fields $w_{\mu \nu}$ decouple, because these fields become either very heavy $\left(M_{s}\right.$ large) or their coupling constant $g_{W}$ becomes very small $(r$ large).

(B) Massless bigravity limit. Second, we consider the massless limit, namely the limit of vanishing Planck mass:

$$
M_{P} \rightarrow 0 \text {. }
$$

It can be realized in string theory by sending the string scale $M_{s}$ to zero: $M_{s} \rightarrow 0$ or equivalently $\alpha^{\prime} \rightarrow \infty$. In this limit, the open string spin-two fields become massless and the bimetric gravity theory becomes conformal. However in string theory this is the tensionless 
limit, where an infinite tower of string states becomes massless in this limit. Therefore the massless bimetric gravity theory only exists as an enormous truncation of higher spin theory with an infinite number of massless higher spin fields. Alternatively, for fixed string scale $M_{s}$, a vanishing Planck mass is obtained by sending $r \rightarrow 0$. Here the size of the internal space becomes much smaller than the string length. Furthermore $g_{W}$ becomes large and the open string spin-two fields $w_{\mu \nu}$ become strongly coupled.

(C) Light spin-two plus massless Yang-Mills limit. Now let us consider the case where the string scale is very small compared to the Planck mass. This is the socalled low string scale scenario, which implies large extra dimensions:

$$
M_{s} \ll M_{P} .
$$

This limit can be achieved by sending $r \rightarrow \infty$, and $M_{W}$ becomes very light compared to $M_{P}$. The spin-two open string fields $w_{\mu \nu}$ become very light, i.e. almost massless, and they are very weakly coupled: $g_{W} \rightarrow 0$. Therefore this limit describes an (almost) conformal field theory on the N D3-branes, with two kinds of open strings: $\mathrm{U}(N)$ Yang-Mills gauge fields and (almost) free spin-two fields $w_{\mu \nu}$. Hence, all fields can be made weakly coupled, and hence this limit is well-defined and feasible. Note that $M_{W} \ll M_{P}$ can be alternatively obtained by keeping $r$ finite, but sending $g_{s} \rightarrow 0$. Then the string theory is weakly coupled and again $g_{W} \rightarrow 0$. Small $g_{s}$ in fact implies that the string scale $M_{s}$ in string units is small compared to the ten-dimensional Planck mass.

\section{Holographic aspects between spin-two on the boundary and spin-four in the bulk}

All open string degrees of freedom/excitations on a D3-brane have a holographic description on $A d S_{5}$. Moreover, the AdS/CFT correspondence is not only true for the massless states, but rather for the entire string modes. We will discuss in this section some aspects of the holography between the first excited open strings, namely the $\mathcal{N}=4$ Weyl multiplet, and the first excited $\mathcal{N}=8$ spin-four supermultiplets of the closed superstring. Holography for $\mathcal{N}=4$ superconformal gravity in the context of $\mathrm{AdS}_{5} / \mathrm{CFT}_{4}$ was already discussed by various authors [48-51], but mainly for non-dynamical superconformal gravitational backgrounds. Here we are dealing with a dynamical spin-two field on the four-dimensional boundary, which will, as we will discuss, correspond to a dynamical spin-four field in the dual five-dimensional bulk theory.

The AdS/CFT correspondence is a duality between open strings on a d-dimensional boundary space and closed strings in a $(\mathrm{d}+1)$-dimensional bulk space. The most famous example is 4 -dimensional $\mathcal{N}=4$ super-Yang-Mills gauge theory located on a stack of $\mathrm{N}$ D3-branes, which is holographically dual to $\mathcal{N}=8$ supergravity on $A d S_{5} \times S^{5}$. Hence for holography to work in general, it is important to consider a limit in string theory, where all closed string modes decouple from the boundary theory. Furthermore we need an (almost) superconformal field theory on the boundary, which possesses the same symmetries as the bulk $A d S_{5}$ background geometry. More precisely, on the boundary we deal with a 
superconformal field theory, with superconformal symmetry group $\mathrm{SU}(2,2 / 4) \times \mathrm{SU}(\mathcal{N})$, where $\mathrm{SU}(4)$ is the R-symmetry group. This agrees with the symmetry group of $\mathcal{N}=8$ supergravity on $A d S_{5}$.

Here we want to describe a possible way, how to include also the open string Weylsupermultiplet $w_{\mu \nu}$ into the $\mathcal{N}=4 \leftrightarrow \mathcal{N}=8$ boundary-bulk holography. Limit A is also not suitable for holography, since closed strings are not decoupled on the brane. Limit A corresponds to the standard AdS/CFT correspondence, namely to the hologrographic duality between the massless spin-one gauge fields on the 4-dimensional boundary and the massless spin-two gravitons in the 5-dimensional bulk. Instead we will focus on the limit B and in particular on the limit $\mathrm{C}$, where closed string gravity on the boundary is decoupled via $M_{P} \rightarrow \infty$, whereas the string scale $M_{s}=M_{W}$ is kept very small compared to the Planck mass, which means that we are considering a large extra volume scenario in string theory. Then the 4-dimensional, non-standard spin-two sector on the boundary possesses an (almost) superconformal symmetry and is supposed to be holographically dual to closed strings in the 5-dimensional $A d S_{5}$ bulk space.

Generally in holography, each field $\phi(x)$ propagating on AdS space is in a one to one correspondence with an operator $\mathcal{O}(x)$ in the field theory, which are coupled together by a term $\int d^{4} x \phi(x) \mathcal{O}(x)$. For a rank $s$ symmetric traceless tensor, there is the following relation between the corresponding mass of the field in the $(d+1)$-dimensional bulk and the scaling dimension $\Delta$ and the spin $s$ of the operator in the conformal field theory on the $d$-dimensional boundary:

$$
m^{2} \alpha^{\prime}=(\Delta+s-2)(\Delta-s+2-d) .
$$

This formula is consistent with the unitarity bound, which is given as

$$
\Delta \geq s-2+d
$$

According to the standard holographic dictionary, the most relevant operator is the conserved boundary energy momentum tensor $T_{\mu}^{\nu}$, which has conformal dimension $\Delta=4$ and spin $s=2$ and hence it saturates the unitarity bound in four dimensions. $T_{\mu}^{\nu}$ is is coupled to a symmetric tensor $g_{\mu \nu}$, which becomes the massless spin-two graviton field in the higher-dimensional bulk theory. ${ }^{4}$ In our concrete case of four-dimensional super YangMills theory plus Weyl ${ }^{2}$ gravity given in eq. (2.34), we can derive the energy momentum tensor from the Yang-Mills action plus the linearized gravity action

$$
S=\int d^{4} x \sqrt{-g}\left(-\frac{1}{4 g_{\mathrm{YM}}^{2}} F_{\mu \nu}^{a} F^{a \mu \nu}+\frac{2}{g_{W}} G_{\mu \nu} w^{\mu \nu}-\left(w_{\mu \nu} w^{\mu \nu}-w^{2}\right)\right) .
$$

However, let us mention that although the original (2.34) theory is invariant under conformal transformations, it seems that (4.3) fails as the Einstein tensor transforms nonhomogeneously. Therefore, in order to restore conformal invariance of (4.3), we have to

\footnotetext{
${ }^{4}$ In case the energy momentum tensor is non-conserved and has dimension $\Delta>2+s$, the corresponding bulk spin-two field becomes massive [53].
} 
assign a non-homogeneous transformation for the field $w_{\mu \nu}$. In fact, it can be verified that under an infinitesimal conformal transformation

$$
\delta g_{\mu \nu}=2 \lambda(x) g_{\mu \nu}
$$

the Einstein tensor transforms as

$$
\delta G_{\mu \nu}=-2 \nabla_{\mu} \nabla_{\nu} \lambda+2 \square \lambda g_{\mu \nu} .
$$

Then, it can be verified [52] that the action (4.3) is invariant if $w_{\mu \nu}$ transforms as

$$
\delta w_{\mu \nu}=-\frac{2}{g_{W}} \nabla_{\mu} \nabla_{\nu} \lambda=\nabla_{\mu} \xi_{\nu}+\nabla_{\nu} \xi_{\mu}
$$

where

$$
\xi_{\mu}=-\frac{1}{g_{W}} \nabla_{\mu} \lambda
$$

In other words, under a conformal transformation, the field $w_{\mu \nu}$ transforms as it would transform under a diffeomorphism generated by the gradient of the conformal factor.

It is straightforward to calculate the energy-momentum tensor for the theory (4.3) which turns out to be

$$
T_{\mu}^{\nu}=T_{F}^{\mu \nu}+T_{w}^{\mu \nu}
$$

where

$$
\begin{aligned}
T_{F}^{\mu \nu}=\frac{1}{g_{\mathrm{YM}}^{2}}\left(F_{\rho}^{a \mu} F^{a \nu \rho}-\frac{1}{4} g^{\mu \nu} F_{\rho \sigma}^{a} F^{a \rho \sigma}\right), \\
T_{w}^{\mu \nu}=\frac{2}{g_{W}}\left\{\square w^{\mu \nu}-\nabla_{\sigma} \nabla^{\nu} w^{\mu \sigma}-\nabla_{\sigma} \nabla^{\mu} w^{\nu \sigma}+R^{\mu \nu} w-R w^{\mu \nu}\right. \\
\quad+\nabla^{\mu} \nabla^{\nu} w+2\left(w^{\mu \rho} w_{\rho}^{\nu}-w w^{\mu \nu}\right)-2\left(G^{\mu}{ }_{\rho} \omega^{\nu \rho}+G^{\nu}{ }_{\rho} \omega^{\mu \rho}\right) \\
\left.\quad+g^{\mu \nu}\left(G_{\rho \sigma} w^{\rho \sigma}-\frac{g_{W}}{2}\left(w_{\rho \sigma} w^{\rho \sigma}-w^{2}\right)+\nabla_{\sigma} \nabla_{\rho} w^{\rho \sigma}-\square w\right)\right\}
\end{aligned}
$$

The equation of motion for $w_{\mu \nu}$ is

$$
w_{\mu \nu}=\frac{2}{g_{W}} S_{\mu \nu}
$$

where $S_{\mu \nu}$ is the Schouten tensor

$$
S_{\mu \nu}=\frac{1}{2}\left(R_{\mu \nu}-\frac{1}{6} R g_{\mu \nu}\right),
$$

and it turns out that $T_{F}^{\mu \nu}$ on-shell is

$$
T_{F}^{\mu \nu}=\frac{16}{g_{W}} B_{\mu \nu},
$$


where

$$
B_{\mu \nu}=\nabla^{\rho} \nabla^{\sigma} W_{\mu \rho \nu \sigma}+\frac{1}{2} R^{\rho \sigma} W_{\mu \rho \nu \sigma}
$$

is the Bach tensor. The latter is symmetric, traceless and divergence-free

$$
B_{\nu}^{\mu}=0, \quad \nabla^{\mu} B_{\mu \nu}=0,
$$

and therefore, $T_{F}^{\mu \nu}$ is also traceless (due to conformal invariance) and divergence-free (due to diff invariance). In addition $B_{\mu \nu}$ transforms under a conformal transformation $g_{\mu \nu} \rightarrow$ $\Omega^{2} g_{\mu \nu}$ as

$$
B_{\nu}^{\mu} \rightarrow \Omega^{-4} B^{\mu}{ }_{\nu}
$$

and therefore it has dimension $\Delta_{B}=4$ (as the energy-momentu tensor).

Next we proceed to the massive spin-four operators on the boundary in dimension $d=4$, which are coupled to massive spin-four, closed string fields in the bulk. In order to be massive their scaling dimension $\Delta$ should be larger than 6 . These fields will become massless in the limit $\alpha^{\prime} \rightarrow \infty$, i.e. $M_{s} \rightarrow 0$. In our concrete case, the relevant spin-four operator $J^{\mu \nu \rho \sigma}$ could be for example

$$
\begin{aligned}
J^{\mu \nu \rho \sigma}= & \mathrm{ST}\left[B^{\mu \nu} B^{\rho \sigma}\right] \\
= & B^{\mu \nu} B^{\rho \sigma}+B^{\rho \nu} B^{\mu \sigma}+B^{\sigma \nu} B^{\rho \mu} \\
& -\frac{1}{2}\left(g^{\mu \rho} B^{\alpha \nu} B_{\alpha}^{\rho}+g^{\mu \sigma} B^{\alpha \nu} B_{\alpha}^{\sigma}+g^{\nu \rho} B^{\alpha \mu} B_{\alpha}^{\rho}+g^{\nu \sigma} B^{\alpha \mu} B_{\alpha}^{\sigma}\right),
\end{aligned}
$$

where ST[] denotes symmetric traceless. Other spin-four operators are

$$
J^{\mu \nu \rho \sigma}=\operatorname{ST}\left[T_{\alpha \beta}^{\mu \nu} T^{\rho \sigma \alpha \beta}\right]
$$

or products of the Weyl tensor, as for example

$$
J^{\mu \nu \rho \sigma}=\mathrm{ST}\left[W^{\mu \alpha \gamma \kappa} W_{\alpha \delta \kappa}^{\rho} W_{\beta \gamma \lambda}^{\nu} W^{\sigma \beta \delta \lambda}\right],
$$

where

$$
\begin{aligned}
T_{\mu \nu \rho \sigma} & =\frac{1}{4}\left(W_{\nu \mu}^{\lambda}{ }^{\kappa} W_{\lambda \sigma \rho \kappa}+\frac{1}{2} \epsilon_{\nu \tau \xi}^{\lambda} \epsilon_{\lambda \sigma}{ }^{\chi \psi} W^{\tau \xi}{ }_{\mu}^{\kappa} W_{\chi \psi \rho \kappa}\right) \\
& =\frac{1}{4}\left(W_{\nu \mu}^{\lambda}{ }^{\kappa} W_{\lambda \sigma \rho \kappa}+W_{\sigma \mu}^{\lambda}{ }^{\kappa} W_{\lambda \nu \rho \kappa}-\frac{1}{2} g_{\nu \sigma} W_{\mu}^{\lambda \tau}{ }^{\kappa} W_{\lambda \tau \sigma \kappa}\right) .
\end{aligned}
$$

is the Bel-Robinson tensor [54-57]. The dimension of the latter is $\Delta_{T}=4$ as under conformal transformations, it transforms as

$$
T_{\rho \sigma}^{\mu \nu} \rightarrow \widehat{T}_{\rho \sigma}^{\mu \nu}=\Omega^{-4} T_{\rho \sigma}^{\mu \nu} .
$$

The operators $J^{\mu \sigma \nu \rho}$ above have spin $s=4$, they transform under conformal transformations as

$$
J_{\nu \rho}^{\mu \sigma} \rightarrow \Omega^{-8} J_{\nu \rho}^{\mu \sigma},
$$


and their dimension is therefore $\Delta_{J}=8$. Hence these operators are then holographically coupled to massive spin-four fields in the bulk, such that we are dealing with a higher spin-four theory in the bulk. In string theory, $J_{\mu \nu \rho \sigma}$ can be viewed as massive composite field with mass square $m^{2}=20 / \alpha^{\prime}$, corresponding to the product of two closed string graviton vertex operators. Note that this mass is the mass on $A d S_{5}$, which is not the same as the mass of the corresponding string state on a flat Minkowski background. In the supersymmetric case, the field content and the supermultiplet structure is precisely as the one given in section 3.2.2, which is obtained by the tensor product of two $\mathcal{N}=4$ super-Weyl multiplets. Since in the decoupling limit, the spin-two fields $w_{\mu \nu}$ are free fields on the $4 \mathrm{D}$ boundary, also the spin-four field in the $A d S_{5}$ bulk space should be a free field, with the following free field equation:

$$
\begin{aligned}
\left(\nabla^{2}+\frac{3}{10} R-m^{2}\right) \Phi_{M N K \Lambda} & =0, \\
\nabla^{M} \Phi_{M N K \Lambda} & =\Phi_{N K \Lambda}^{M}=0, \quad M, N, \cdots=0,1, \cdots 4,
\end{aligned}
$$

where $R$ is the scalar curvature of the $A d S_{5}$ space. $^{5}$

So in summary, the Yang-Mills energy momentum tensor $T_{\mu \nu}$ couples to a spin-two field in the bulk, the standard graviton on $A d S_{5} \times S^{5}$, whereas $J_{\mu \nu \rho \sigma}$ couples to a spin-four field in the bulk. It means in particular when considering just the $\mathcal{N}$-extended (Weyl) ${ }^{2}$ supergravity theory in four dimensions without the Yang-Mills part that this theory is the holographically dual boundary theory of an $A d S_{5}$ bulk theory, which is a higher spin theory with a spin-four multiplet of the $2 \mathcal{N}$-extended supersymmetry algebra in five dimensions. These kind of theories, denoted by W-supergravities, were recently constructed [47] in flat four-dimensional space-time using a double copy construction. Therefore, the (almost) massless spin-two fields $w_{\mu \nu}$ are conjectured to be dual to $\mathcal{N}=8$ spin-four fields on $A d S_{5} \times$ $S^{5}$. To support this conjecture it would be important to compute some correlation functions of $J_{\mu \nu \rho \sigma}$ on the boundary and compare them with the corresponding spin-four correlation functions in the bulk.

\section{Conclusions}

In this paper we have discussed a special version of $\mathcal{N}=4$ supersymmetric bimetric gravity coupled to $\mathcal{N}=4$ super Yang-Mills gauge theory. We have argued that, just like the open string Yang-Mills gauge fields, the massive spin-two graviton supermultiplet originates from open string excitations on D3-branes and hence is localized in four space-time dimensions,

\footnotetext{
${ }^{5}$ In general, a spin-s field in $(A) d S_{d}$ is described by a totally symmetric, traceless and divergentless tensor $\Phi_{M_{1} \cdots M_{s}}$ and obeys the equation [58, 59]

$$
\begin{aligned}
{\left[\left(\nabla^{2}+\left(s^{2}+s(d-6)+6-2 d\right) \frac{R}{d(d-1)}-m^{2}\right] \Phi_{M_{1} \cdots M_{s}}\right.} & =0, \\
\nabla^{M_{1}} \Phi_{M_{1} \cdots M_{s}}=\Phi_{M_{1} \cdots M_{s}}^{M_{1}} & =0, \quad M_{i} \cdots=0,1, \cdots d-1 .
\end{aligned}
$$
}


whereas the standard massless spin-two graviton supermultiplet is coming from the closed string sector. We then argued that effective action of this bimetric theory is given by the four-derivative, $\mathcal{N}=4$ supersymmetric $\mathrm{Weyl}^{2}$ action, whose Weyl-supermultiplet precisely embraces the same number of degrees of freedom as the first massive open string excitations on the D3-branes. In the massless limit, where the mass of the open string "gravitons" and their superpartners go to zero, the theory becomes $\mathcal{N}=4$ superconformal. We discussed that the holographic description of this quadratic spin-two superconformal gravity on the four-dimensional boundary is given in terms of a higher $\mathcal{N}=8$ spin-four theory in the $A d S_{5}$ bulk space. We have constructed the corresponding $\mathcal{N}=8$ spin-four supermultiplet in terms of massive closed string excitations in four space-time dimensions, which then can be lifted to the five-dimensional $A d S_{5}$ space. In addition we have identified certain spinfour operators on the four-dimensional boundary space, which, following the holographic dictionary, can couple to the spin-four fields in the five-dimensional bulk.

At the end of the paper, we like to close with the following additional remarks:

- It is clear from string theory that the massive open string spin-two state cannot be a ghost state. So eventually one has to write down an effective action for this spin-two state, which is ghost-free. But here we are truncating the spectrum to the first excited level and neglecting all the higher open string excitations. In the same way we are restricting the effective action to be just with four derivatives, but we neglect all the higher derivative interactions [60]. It is now still a conjecture that the full effective action action of this open string spin-two state can be written as an infinite power series expansion of the Weyl-tensor. In fact it was recently argued in [31] that adding an infinite series of curvature tensors should provide an action which propagated a ghost-free open string spin-two particle. Truncating this series to $\mathrm{Weyl}^{2}$, the spin-two particle becomes a ghost.

- In case we are dealing with a stack of $\mathrm{N}$ D3-branes, the massive $\mathcal{N}=4$ Weyl supermultiplet is colored, just like the $\mathrm{U}(N)$ gauge fields. Therefore one is dealing with $N^{2}$ copies of interacting spin-two Weyl supermuliplets. In this paper we have considered the simpler case of just one single, neutral Weyl supermuliplet, which belongs to the $\mathrm{U}(1)$ part of the $\mathrm{U}(N)$ symmetry group, or simply is the relevant open string excitation for the case $N=1$. For an U(1) open string one might expect as effective action the full tower of $\alpha^{\prime}$ terms of the Born-Infeld action, but at the two derivative level the effective action is still given by the Maxwell plus conformal gravity action.

- It would be interesting to compute the string scattering amplitudes between the massless and massive string fields using techniques already applied in [45] in order to confirm the effective Weyl ${ }^{2}$ action and the couplings between the Yang-Mills and the Weyl sectors, proposed in this paper. ${ }^{6}$

\footnotetext{
${ }^{6}$ As discussed with the referee of this paper, the string amplitudes are apparently matched by the action given in eq. (2.59) of conformal supergravity in twistor-string theory [42]. For constant function $\mathcal{H}\left(\phi_{\alpha}\right)$ this is of course nothing else than the standard Weyl square action, which basically confirms in this way the results of the paper. We are grateful to the referee for drawing our attention to this point.
} 
- The massive closed string spin-four field can be viewed as a kind of a bound state of two massive open string spin-two states, in analogy to the massless closed string graviton, which can be regarded as the bound state of two massless open string gauge bosons. This observation relies in the structure of the string vertex operators and is also the basis of the double copy constructions, which was recently also worked out four the spin-four case [47].

- It should be possible to perform a socalled S-fold projection, getting completely get rid off the massless Yang-Mills sector. In this case one would entirely deal with strongly coupled, massive Weyl ${ }^{2}$ supergravity on the boundary and with massive spin-four supergravity in the bulk, a theory denoted by W-supergravity, recently constructed in [47]. In the massless, superconformal limit, the spin-four W-supergravity on $A d S_{5}$ also becomes massless.

- The scalar potential should capture also the solutions which are not the one of Einstein supergravity. In the bosonic case these are the solutions where the Bach tensor vanishes but not the Ricci tensor. While the first break conformal to Poincare supergravity, the others may also break supersymmetry even partially, which still has to be discovered yet. It is likely that any conformally flat space is a solution with vanishing Bach tensor so it is conceivable that AdS or even dS space are solutions of massless Weyl supergravity, as it is true in the simplest bosonic case.

- Finally, we would like to stress that the superconformal symmetry of the supersymmetric Weyl ${ }^{2}$ theory is a classical symmetry. ${ }^{7}$ Althought such theories are powercounting renormalizable, their one-loop beta-functions are be non-vanishing [62] and therefore they suffer from a conformal anomaly. The latter leads to serious problems since conformal symmetry is gauged in Weyl gravity and therefore leads to inconsistencies [63-65]. The same conclusion can be drawn by considering the chiral gauge anomalies of the $\mathrm{SU}(4)$ R-symmetry [66] and recalling that all anomalies are accommodated in the same multiplet of the $\mathcal{N}=4$ superconformal symmetry.

\section{Acknowledgments}

We like to thank Costas Bachas, Elias Kiritsis, Stefan Theisen and Timo Weigand for useful discussions. Furthermore we gratefully knowledge enlightening discussions with Augusto Sagnotti on the Scalar Potential of Massive Weyl Supergravity and with Angnis SchmidtMay on Bimetric Gravity. The work of S.F. is supported in part by CERN TH Department and INFN-CSN4-GSS. The work of D.L. is supported by the ERC Advanced Grant "Strings and Gravity" (Grant No. 320045) and the Excellence Cluster Universe. A.K. is supported by the GSRT under the EDEIL/67108600.

\footnotetext{
${ }^{7}$ It has been conjectured in [61] that $\mathcal{N}=4$ Poincaré supergravity has also a hidden superconformal symmetry.
} 
Open Access. This article is distributed under the terms of the Creative Commons Attribution License (CC-BY 4.0), which permits any use, distribution and reproduction in any medium, provided the original author(s) and source are credited.

\section{References}

[1] K.S. Stelle, Renormalization of higher derivative quantum gravity, Phys. Rev. D 16 (1977) 953 [INSPIRE].

[2] K.S. Stelle, Classical gravity with higher derivatives, Gen. Rel. Grav. 9 (1978) 353 [INSPIRE].

[3] D.G. Boulware, G.T. Horowitz and A. Strominger, Zero energy theorem for scale invariant gravity, Phys. Rev. Lett. 50 (1983) 1726 [INSPIRE].

[4] F. David and A. Strominger, On the calculability of Newton's constant and the renormalizability of scale invariant quantum gravity, Phys. Lett. 143B (1984) 125 [INSPIRE].

[5] G.T. Horowitz, Quantum cosmology with a positive definite action, Phys. Rev. D 31 (1985) 1169 [INSPIRE].

[6] S. Deser and B. Tekin, Shortcuts to high symmetry solutions in gravitational theories, Class. Quant. Grav. 20 (2003) 4877 [gr-qc/0306114] [INSPIRE].

[7] S. Deser and B. Tekin, New energy definition for higher curvature gravities, Phys. Rev. D 75 (2007) 084032 [gr-qc/0701140] [INSPIRE].

[8] G. 't Hooft, A class of elementary particle models without any adjustable real parameters, Found. Phys. 41 (2011) 1829 [arXiv:1104.4543] [INSPIRE].

[9] J. Maldacena, Einstein gravity from conformal gravity, arXiv:1105.5632 [INSPIRE].

[10] H. Lü, C.N. Pope, E. Sezgin and L. Wulff, Critical and non-critical Einstein-Weyl supergravity, JHEP 10 (2011) 131 [arXiv:1107.2480] [INSPIRE].

[11] S.F. Hassan and R.A. Rosen, Bimetric gravity from ghost-free massive gravity, JHEP 02 (2012) 126 [arXiv:1109.3515] [InSPIRE].

[12] S.F. Hassan, R.A. Rosen and A. Schmidt-May, Ghost-free massive gravity with a general reference metric, JHEP 02 (2012) 026 [arXiv: 1109.3230] [INSPIRE].

[13] S.F. Hassan, A. Schmidt-May and M. von Strauss, Higher derivative gravity and conformal gravity from bimetric and partially massless bimetric theory, Universe 1 (2015) 92 [arXiv: 1303.6940] [INSPIRE].

[14] S. Deser, E. Joung and A. Waldron, Gravitational- and self- coupling of partially massless spin 2, Phys. Rev. D 86 (2012) 104004 [arXiv:1301.4181] [InSPIRE].

[15] F. Del Monte, D. Francia and P.A. Grassi, Multimetric supergravities, JHEP 09 (2016) 064 [arXiv: 1605.06793] [INSPIRE].

[16] S. Ferrara, A. Kehagias and D. Lüst, Aspects of Weyl supergravity, JHEP 08 (2018) 197 [arXiv: 1806.10016] [INSPIRE].

[17] S. Ferrara, M. Kaku, P.K. Townsend and P. van Nieuwenhuizen, Gauging the graded conformal group with unitary internal symmetries, Nucl. Phys. B 129 (1977) 125 [INSPIRE].

[18] M. Kaku, P.K. Townsend and P. van Nieuwenhuizen, Superconformal unified field theory, Phys. Rev. Lett. 39 (1977) 1109 [INSPIRE]. 
[19] M. Kaku, P.K. Townsend and P. van Nieuwenhuizen, Properties of conformal supergravity, Phys. Rev. D 17 (1978) 3179 [INSPIRE].

[20] D.Z. Freedman and A. Van Proeyen, Supergravity, Cambridge University Press, Cambridge U.K. (2012).

[21] E.S. Fradkin and A.A. Tseytlin, Conformal supergravity, Phys. Rept. 119 (1985) 233 [INSPIRE].

[22] B. de Wit and S. Ferrara, On higher order invariants in extended supergravity, Phys. Lett. B 81 (1979) 317.

[23] D. Lüst, S. Stieberger and T.R. Taylor, The LHC string hunter's companion, Nucl. Phys. B 808 (2009) 1 [arXiv:0807.3333] [INSPIRE].

[24] L. Álvarez-Gaumé et al., Aspects of quadratic gravity, Fortsch. Phys. 64 (2016) 176 [arXiv: 1505.07657] [INSPIRE].

[25] A. Salvio, Quadratic gravity, Front. in Phys. 6 (2018) 77 [arXiv: 1804.09944] [INSPIRE].

[26] P.K. Townsend and P. van Nieuwenhuizen, Simplifications of conformal supergravity, Phys. Rev. D 19 (1979) 3166 [INSPIRE].

[27] S. Cecotti, S. Ferrara, M. Porrati and S. Sabharwal, New minimal higher derivative supergravity coupled to matter, Nucl. Phys. B 306 (1988) 160 [INSPIRE].

[28] S. Ferrara and M. Villasante, Curvatures, Gauss-Bonnet and Chern-Simons multiplets in old minimal $N=1$ supergravity, J. Math. Phys. 30 (1989) 104 [InSPIRE].

[29] F. Farakos, S. Ferrara, A. Kehagias and D. Lüst, Non-linear realizations and higher curvature supergravity, Fortsch. Phys. 65 (2017) 1700073 [arXiv:1707.06991] [INSPIRE].

[30] E.A. Bergshoeff, O. Hohm and P.K. Townsend, Massive gravity in three dimensions, Phys. Rev. Lett. 102 (2009) 201301 [arXiv:0901.1766] [INSPIRE].

[31] B. Gording and A. Schmidt-May, Ghost-free infinite derivative gravity, JHEP 09 (2018) 044 [Erratum ibid. 1810 (2018) 115] [arXiv:1807.05011] [INSPIRE].

[32] S. Ferrara and B. Zumino, Structure of conformal supergravity, Nucl. Phys. B 134 (1978) 301 [INSPIRE].

[33] S. Ferrara and B. Zumino, Transformation properties of the supercurrent, Nucl. Phys. B 87 (1975) 207 [INSPIRE].

[34] S. Ferrara, M.T. Grisaru and P. van Nieuwenhuizen, Poincaré and conformal supergravity models with closed algebras, Nucl. Phys. B 138 (1978) 430 [INSPIRE].

[35] R.J. Riegert, The particle content of linearized conformal gravity, Phys. Lett. A 105 (1984) 110 [INSPIRE].

[36] S.F. Hassan, A. Schmidt-May and M. von Strauss, On partially massless bimetric gravity, Phys. Lett. B 726 (2013) 834 [arXiv:1208.1797] [InSPIRE].

[37] E. Bergshoeff, M. de Roo and B. de Wit, Extended conformal supergravity, Nucl. Phys. B 182 (1981) 173 [INSPIRE].

[38] J.P. Derendinger and S. Ferrara, $N=1$ and $N=2$ supergravities coupled to matter: superhiggs effect and geometrical structure, in Supersymmetry and Supergravity 1984, B. de Wit et al. eds., World Scientific, Singapore (1984).

[39] M. de Roo, Matter coupling in $N=4$ supergravity, Nucl. Phys. B 255 (1985) 515 [inSPIRE]. 
[40] M. de Roo, Gauged N=4 matter couplings, Phys. Lett. B 156 (1985) 331.

[41] D. Butter, F. Ciceri, B. de Wit and B. Sahoo, Construction of all $N=4$ conformal supergravities, Phys. Rev. Lett. 118 (2017) 081602 [arXiv: 1609.09083] [INSPIRE].

[42] N. Berkovits and E. Witten, Conformal supergravity in twistor-string theory, JHEP 08 (2004) 009 [hep-th/0406051] [INSPIRE].

[43] S. Ferrara, C.A. Savoy and B. Zumino, General massive multiplets in extended supersymmetry, Phys. Lett. B 100 (1981) 393.

[44] H. Johansson, G. Mogull and F. Teng, Unraveling conformal gravity amplitudes, JHEP 09 (2018) 080 [arXiv: 1806.05124] [INSPIRE].

[45] W.-Z. Feng et al., Direct production of lightest Regge resonances, Nucl. Phys. B 843 (2011) 570 [arXiv: 1007.5254] [INSPIRE].

[46] R. Blumenhagen, D. Lüst and S. Theisen, Basic concepts of string theory, Springer, Germany (2012).

[47] S. Ferrara and D. Lüst, Spin-four $\mathcal{N}=7 \mathrm{~W}$-supergravity: S-fold and double copy construction, JHEP 07 (2018) 114 [arXiv: 1805.10022] [INSPIRE].

[48] H. Liu and A.A. Tseytlin, $D=4$ superYang-Mills, $D=5$ gauged supergravity and $D=4$ conformal supergravity, Nucl. Phys. B 533 (1998) 88 [hep-th/9804083] [INSPIRE].

[49] M. Henningson and K. Skenderis, The holographic Weyl anomaly, JHEP 07 (1998) 023 [hep-th/9806087] [INSPIRE].

[50] V. Balasubramanian, E.G. Gimon, D. Minic and J. Rahmfeld, Four-dimensional conformal supergravity from AdS space, Phys. Rev. D 63 (2001) 104009 [hep-th/0007211] [InSPIRE].

[51] M. Abou-Zeid, Actions for curved branes, hep-th/0001127 [INSPIRE].

[52] S. Deser and A. Waldron, Gauge invariances and phases of massive higher spins in (A)dS, Phys. Rev. Lett. 87 (2001) 031601 [hep-th/0102166] [INSPIRE].

[53] C. Bachas and I. Lavdas, Massive Anti-de Sitter gravity from string theory, JHEP 11 (2018) 003 [arXiv: 1807.00591] [INSPIRE].

[54] L. Bel, Sur la radiation gravitationelle, C. R. Math. Acad. Sci. Paris 247 (1958) 1094.

[55] L. Bel, Introduction d'un tenseur du quartieme order, C. R. Math. Acad. Sci. Paris 248 (1959) 1297.

[56] I. Robinson, unpublished King's College lectures, London, U.K. (1958).

[57] I. Robinson, On the Bel-Robinson tensor, Class. Quant. Grav. 14 (1997) 4331

[58] S. Deser and A. Waldron, Arbitrary spin representations in de Sitter from dS/CFT with applications to dS supergravity, Nucl. Phys. B 662 (2003) 379 [hep-th/0301068] [INSPIRE].

[59] R.R. Metsaev, Massive totally symmetric fields in AdS(d), Phys. Lett. B 590 (2004) 95 [hep-th/0312297] [INSPIRE].

[60] S. Weinberg, Effective field theory for inflation, Phys. Rev. D 77 (2008) 123541 [arXiv: 0804.4291] [INSPIRE].

[61] S. Ferrara, R. Kallosh and A. Van Proeyen, Conjecture on hidden superconformal symmetry of $N=4$ supergravity, Phys. Rev. D 87 (2013) 025004 [arXiv: 1209.0418] [INSPIRE]. 
[62] E.S. Fradkin and A.A. Tseytlin, One loop $\beta$-function in conformal supergravities, Nucl. Phys. B 203 (1982) 157 [INSPIRE].

[63] D.M. Capper and M.J. Duff, Conformal anomalies and the renormalizability problem in quantum gravity, Phys. Lett. A 53 (1975) 361 [INSPIRE].

[64] E.S. Fradkin and A.A. Tseytlin, Conformal anomaly in Weyl theory and anomaly free superconformal theories, Phys. Lett. B 134 (1984) 187.

[65] A.A. Tseytlin, On divergences in non-minimal $N=4$ conformal supergravity, J. Phys. A 50 (2017) 48LT01 [arXiv:1708.08727] [INSPIRE].

[66] H. Römer and P. van Nieuwenhuizen, Axial anomalies in $N=4$ conformal supergravity, Phys. Lett. B 162 (1985) 290. 